

\title{
Noetherianity for infinite-dimensional toric varieties
}

\author{
Jan Draisma, Rob Eggermont, Robert Krone and Anton Leykin
}

\begin{abstract}
We consider a large class of monomial maps respecting an action of the infinite symmetric group, and prove that the toric ideals arising as their kernels are finitely generated up to symmetry. Our class includes many important examples where Noetherianity was recently proved or conjectured. In particular, our results imply Hillar-Sullivant's independent set theorem and settle several finiteness conjectures due to Aschenbrenner, Martín del Campo, Hillar, and Sullivant.

We introduce a matching monoid and show that its monoid ring is Noetherian up to symmetry. Our approach is then to factorize a more general equivariant monomial map into two parts going through this monoid. The kernels of both parts are finitely generated up to symmetry: recent work by Yamaguchi-OgawaTakemura on the (generalized) Birkhoff model provides an explicit degree bound for the kernel of the first part, while for the second part the finiteness follows from the Noetherianity of the matching monoid ring.
\end{abstract}

\section{Introduction and main result}

Families of algebraic varieties parameterized by combinatorial data arise in various areas of mathematics, such as statistics (e.g., phylogenetic models parameterized by trees [Allman and Rhodes 2008; Draisma and Kuttler 2009; Draisma and Eggermont 2015; Pachter and Sturmfels 2005] or the relations among path probabilities in Markov chains parameterized by path length [Haws et al. 2014; Norén 2015]), commutative algebra (e.g., Segre powers of a fixed vector space parameterized by the exponent [Snowden 2013] or Laurent lattice ideals [Hillar and Martín del Campo 2013]), and combinatorics (e.g., algebraic matroids arising from determinantal ideals parameterized by matrix sizes [Kiraly and Rosen 2013] or edge ideals of hypergraphs parameterized by the number of vertices [Gross and Petrović 2013]).

Draisma and Eggermont are supported by a Vidi grant from the Netherlands Organisation for Scientific Research (NWO) .

Krone and Leykin are supported in part by the NSF under grant DMS 1151297.

MSC2010: primary 13E05; secondary 13P10, 14M25.

Keywords: Noetherianity up to symmetry, binomial ideals. 
A natural question is whether such families stabilize as some of the combinatorial data tend to infinity. A recently established technique for proving such stabilization is passing to an infinite-dimensional limit of the family, giving some equations for that limit, and showing that those equations cut out a suitably Noetherian space. This then implies that the limit itself is given by finitely many further equations, and that the family stabilizes. This technique is applied, for instance, in the proof of the independent set theorem [Hillar and Sullivant 2012], and in the first author's work on the Gaussian $k$-factor model, chirality varieties, and tensors of bounded rank [Draisma 2010; Draisma and Kuttler 2014].

In the present paper, we follow a similar approach, utilizing the new concept of a matching monoid to prove that stabilization happens for a large class of toric varieties. Our main theorem provides one-step proofs for several existing results that were established in a rather less general context; and it settles conjectures and questions from [Aschenbrenner and Hillar 2007; Hillar and Sullivant 2012; Hillar and Martín del Campo 2013]. There is a list of three such consequences at the end of this Introduction. Moreover, we show Noetherianity in a constructive manner by complementing the main theorem with an algorithm that produces a finite set of equations whose orbits define the infinite-dimensional toric variety under consideration.

Instead of working with inverse systems of affine varieties, we work directly with direct limits of their coordinate rings. In fact, we formulate our main theorem directly in the infinite-dimensional setting, as going back to families of finitedimensional coordinate rings of toric varieties is fairly straightforward. Throughout, $\mathbb{N}$ denotes $\{0,1,2,3, \ldots\}$, and for $k \in \mathbb{N}$ we write $[k]:=\{0, \ldots, k-1\}$. We write $\operatorname{Sym}(\mathbb{N})$ for the group of all bijections $\mathbb{N} \rightarrow \mathbb{N}$, and $\operatorname{Inc}(\mathbb{N})$ for the monoid of all strictly increasing maps $\mathbb{N} \rightarrow \mathbb{N}$. Let $Y$ be a set equipped with an action of $\operatorname{Sym}(\mathbb{N})$. We require that the action has the following property: for each $y \in Y$ there exists a $k_{y} \in \mathbb{N}$ such that $y$ is fixed by all of $\operatorname{Sym}\left(\mathbb{N} \backslash\left[k_{y}\right]\right)$, i.e., by all elements of $\operatorname{Sym}(\mathbb{N})$ that fix $\left[k_{y}\right]$ elementwise. In this setting, $\operatorname{Inc}(\mathbb{N})$ also acts on $Y$, as follows: for $\pi \in \operatorname{Inc}(\mathbb{N})$ and $y \in Y$, choose a $\pi^{\prime} \in \operatorname{Sym}(\mathbb{N})$ that agrees with $\pi$ on $\left[k_{y}\right]$, set $\pi y:=\pi^{\prime} y$, and observe that this does not depend on the choice of $\pi^{\prime}$. Observe that for each $y \in Y$ the $\operatorname{Inc}(\mathbb{N})$-orbit $\operatorname{Inc}(\mathbb{N}) y$ is contained in $\operatorname{Sym}(\mathbb{N}) y$, and that the latter is in fact equal to the orbit of $y$ under the countable subgroup of $\operatorname{Sym}(\mathbb{N})$ consisting of permutations fixing all but finitely many natural numbers. See also [Hillar and Sullivant 2012, Section 5].

Let $R$ be a Noetherian ring (commutative, with 1 ), and let $R[Y]$ be the commutative $R$-algebra of polynomials in which the elements of $Y$ are the variables and the coefficients come from $R$. The group $\operatorname{Sym}(\mathbb{N})$ acts by $R$-algebra automorphisms on $R[Y]$ by permuting the variables. Furthermore, let $k$ be a natural number, and let $Z=\left\{z_{i j} \mid i \in[k], j \in \mathbb{N}\right\}$ be a second set of variables, with a $\operatorname{Sym}(\mathbb{N})$-action given 
by $\pi z_{i j}=z_{i \pi(j)}$. Extend this action to an action by $R$-algebra automorphisms of $R[Z]$. Note that the $\operatorname{Sym}(\mathbb{N})$-actions on $R[Y], Z$, and $R[Z]$ all have the property required of the action on $Y$. Hence they also yield $\operatorname{Inc}(\mathbb{N})$-actions, by means of injective $R$-algebra endomorphisms in the case of $R[Y]$ and $R[Z]$. In general, when a monoid $\Pi$ acts on a ring $S$ by means of endomorphisms, $S$ is called $\Pi$-Noetherian if every $\Pi$-stable ideal in $S$ is generated by the union of finitely many $\Pi$-orbits of elements, i.e., if $S$ is Noetherian as a module under the skew monoid ring $S * \Pi$; see [Hillar and Sullivant 2012].

Theorem 1.1 (main theorem). Assume that $\operatorname{Sym}(\mathbb{N})$ has only finitely many orbits on $Y$. Let $\varphi: R[Y] \rightarrow R[Z]$ be a $\operatorname{Sym}(\mathbb{N})$-equivariant homomorphism that maps each $y \in Y$ to a monomial in the $z_{i j}$. Then $\operatorname{ker} \varphi$ is generated by finitely many $\operatorname{Inc}(\mathbb{N})$-orbits of binomials, and $\operatorname{im} \varphi \cong R[Y] / \operatorname{ker} \varphi$ is an $\operatorname{Inc}(\mathbb{N})$-Noetherian ring.

If an ideal is $\operatorname{Sym}(\mathbb{N})$-stable, then it is certainly $\operatorname{Inc}(\mathbb{N})$-stable, so the last statement implies that $R[Y] / \operatorname{ker} \varphi$ is $\operatorname{Sym}(\mathbb{N})$-Noetherian. The conditions in the theorem are sharp in the following senses.

(1) The ring $R[Y]$ itself is typically not $\operatorname{Sym}(\mathbb{N})$-Noetherian, let alone $\operatorname{Inc}(\mathbb{N})$ Noetherian. Take, for instance, $Y=\left\{y_{i j} \mid i, j \in \mathbb{N}\right\}$ with $\operatorname{Sym}(\mathbb{N})$ acting diagonally on both indices, and take any $R$ with $1 \neq 0$. Then the $\operatorname{Sym}(\mathbb{N})$-orbits of the monomials

\section{$y_{12} y_{21}, y_{12} y_{23} y_{31}, y_{12} y_{23} y_{34} y_{41}, \ldots$}

generate a $\operatorname{Sym}(\mathbb{N})$-stable ideal that is not generated by any finite union of orbits (see [Aschenbrenner and Hillar 2007, Proposition 5.2]).

(2) The $R$-algebra $R[Z]$ is $\operatorname{Sym}(\mathbb{N})$-Noetherian, and even $\operatorname{Inc}(\mathbb{N})$-Noetherian [Cohen 1987; Hillar and Sullivant 2012] — this is the special case of our theorem where $Y=Z$ and $\varphi$ is the identity - but $\operatorname{Sym}(\mathbb{N})$-stable subalgebras of $R[Z]$ need not be, even when generated by finitely many $\operatorname{Sym}(\mathbb{N})$-orbits of polynomials. For instance, an (as yet) unpublished theorem due to Krasilnikov says that in characteristic 2, the ring generated by all $2 \times 2$-minors of a $2 \times \mathbb{N}$-matrix of variables is $\operatorname{not} \operatorname{Sym}(\mathbb{N})$ Noetherian. Put differently, we do not know if the finite-generatedness of $\operatorname{ker} \varphi$ in the main theorem continues to hold if $\varphi$ is an arbitrary $\operatorname{Sym}(\mathbb{N})$-equivariant homomorphism, but certainly the quotient is not, in general, $\operatorname{Sym}(\mathbb{N})$-Noetherian.

(3) Moreover, subalgebras of $R[Z]$ generated by finitely many $\operatorname{Inc}(\mathbb{N})$-orbits of monomials need not be Inc $(\mathbb{N})$-Noetherian; see Krasilnikov's example in [Hillar and Sullivant 2012]. However, our main theorem implies that subalgebras of $R[Z]$ generated by finitely many $\operatorname{Sym}(\mathbb{N})$-orbits of monomials are $\operatorname{Inc}(\mathbb{N})$-Noetherian.

Our main theorem applies to many problems on Markov bases of families of point sets. In such applications, the following strengthening is sometimes useful. 
Corollary 1.2. Assume that $\operatorname{Sym}(\mathbb{N})$ has only finitely many orbits on $Y$, and let $S$ be an $R$-algebra with trivial $\operatorname{Sym}(\mathbb{N})$-action. Let $\varphi: R[Y] \rightarrow S[Z]$ be a $\operatorname{Sym}(\mathbb{N})$ equivariant $R$-algebra homomorphism that maps each $y \in Y$ to an element of $S$ times a monomial in the $z_{i j}$. Then $\operatorname{ker} \varphi$ is generated by finitely many $\operatorname{Inc}(\mathbb{N})$-orbits of binomials, and $\operatorname{im} \varphi \cong R[Y] / \operatorname{ker} \varphi$ is an $\operatorname{Inc}(\mathbb{N})$-Noetherian ring.

Proof of the corollary given the main theorem. Let $y_{p}, p \in[N]$ be representatives of the $\operatorname{Sym}(\mathbb{N})$-orbits on $Y$. Then for all $p \in[N]$ and $\pi \in \operatorname{Sym}(\mathbb{N})$ we have $\varphi\left(\pi y_{p}\right)=s_{p} \pi u_{p}$ for some monomial $u_{p}$ in the $z_{i j}$ and some $s_{p}$ in $S$. Apply the main theorem to $Y^{\prime}:=Y \times \mathbb{N}$ and $Z \cup Z^{\prime}$ with $Z^{\prime}:=\left\{z_{p, j}^{\prime} \mid p \in[N], j \in \mathbb{N}\right\}$ and $\varphi^{\prime}$ the map that sends the variable $\left(\pi y_{p}, j\right)$ to $z_{p, j}^{\prime} \pi u_{p}$. Consider the commutative diagram

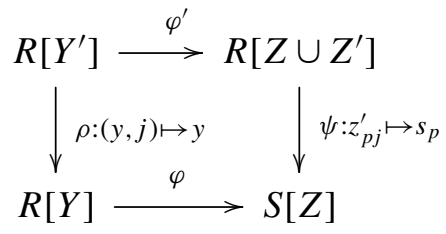

of $\operatorname{Sym}(\mathbb{N})$-equivariant $R$-algebra homomorphisms. By the main theorem, im $\varphi^{\prime}$ is $\operatorname{Inc}(\mathbb{N})$-Noetherian, hence so is its image under $\psi$; and this image equals $\operatorname{im} \varphi$ because $\rho$ is surjective. Similarly, $\operatorname{ker}\left(\psi \circ \varphi^{\prime}\right)$ is generated by finitely many $\operatorname{Inc}(\mathbb{N})$ orbits (because this is the case for both $\operatorname{ker} \varphi^{\prime}$ and $\left.\operatorname{ker} \psi\right|_{\operatorname{im} \varphi^{\prime}}$ ), hence so is its image under $\rho$; and this image is $\operatorname{ker} \varphi$ because $\rho$ is surjective.

Here are some consequences of our main theorem.

(1) Our main theorem implies [Aschenbrenner and Hillar 2007, Conjecture 5.10], to the effect that chains of ideals arising as kernels of monomial maps of the form $y_{i_{1}, \ldots, i_{k}} \mapsto z_{i_{1}}^{a_{1}} \cdots z_{i_{k}}^{a_{k}}$, where the indices $i_{1}, \ldots, i_{k}$ are required to be distinct, stabilize. Aschenbrenner and Hillar proved this in the squarefree case, where the $a_{j}$ are equal to 1 . In the Laurent polynomial setting more is known [Hillar and Martín del Campo 2013].

(2) A consequence of [de Loera et al. 1995] is that for any $n \geq 4$ the vertex set $\left\{v_{i j}:=e_{i}+e_{j} \mid i \neq j\right\} \subseteq \mathbb{R}^{n}$ of the $(n-1)$-dimensional second hypersimplex has a Markov basis corresponding to the relations $v_{i j}=v_{j i}$ and $v_{i j}+v_{k l}=v_{i l}+v_{k j}$. Here is a qualitative generalization of this fact. Let $m$ and $k$ be fixed natural numbers. For every $n \in \mathbb{N}$ consider a finite set $P_{n} \subseteq \mathbb{Z}^{m} \times \mathbb{Z}^{k \times n}$. Let $\operatorname{Sym}(n)$ act trivially on $\mathbb{Z}^{m}$ and by permuting columns on $\mathbb{Z}^{k \times n}$. Assume that there exists an $n_{0}$ such that $\operatorname{Sym}(n) P_{n_{0}}=P_{n}$ for $n \geq n_{0}$; here we think of $\mathbb{Z}^{k \times n_{0}}$ as the subset of $\mathbb{Z}^{k \times n}$ where the last $n-n_{0}$ columns are zero. Then Corollary 1.2 implies that there exists an $n_{1}$ such that for any Markov basis $M_{n_{1}}$ for the relations among the points in $P_{n_{1}}$, $\operatorname{Sym}(n) M_{n_{1}}$ is a Markov basis for $P_{n}$ for all $n \geq n_{1}$. For the second hypersimplex, $n_{0}$ equals 2 and $n_{1}$ equals 4 . 
(3) A special case of the previous consequence is the independent set theorem of [Hillar and Sullivant 2012]. We briefly illustrate how to derive it directly from Corollary 1.2. Let $m$ be a natural number and let $\mathcal{F}$ be a family of subsets of a finite set $[m]$. Let $T$ be a subset of $[m]$ and assume that each $F \in \mathcal{F}$ contains at most one element of $T$. In other words, $T$ is an independent set in the hypergraph determined by $\mathcal{F}$. For $t \in[m] \backslash T$ let $r_{t}$ be a natural number. Set $Y:=\left\{y_{\alpha} \mid \alpha \in \mathbb{N}^{T} \times \prod_{t \in[m] \backslash T}\left[r_{t}\right]\right\}$ and $Z:=\left\{z_{F, \alpha} \mid F \in \mathcal{F}, \alpha \in \mathbb{N}^{F \cap T} \times \prod_{F \backslash T}\left[r_{t}\right]\right\}$, and let $\varphi$ be the homomorphism $\mathbb{Z}[Y] \rightarrow \mathbb{Z}[Z]$ that maps $y_{\alpha}$ to $\prod_{F \in \mathcal{F}} z_{F,\left.\alpha\right|_{F}}$, where $\left.\alpha\right|_{F}$ is the restriction of $\alpha$ from $[m]$ to $F$. Then $\varphi$ is equivariant with respect to the action of $\operatorname{Sym}(\mathbb{N})$ on the variables induced by the diagonal action of $\operatorname{Sym}(\mathbb{N})$ on $\mathbb{N}^{T}$, and (a strong form of) the independent set theorem boils down to the statement that $\operatorname{ker} \varphi$ is generated by finitely many $\operatorname{Sym}(\mathbb{N})$-orbits of binomials. By the condition that $T$ is an independent set, each $z$-variable has at most one index running through all of $\mathbb{N}$. Setting $S$ to be $\mathbb{Z}\left[z_{F, \alpha} \mid F \cap T=\varnothing\right]$, we find that $Y, S$, the remaining $z_{F, \alpha}$-variables, with $|F \cap T|=1$, and the map $\varphi$ satisfy the conditions of the corollary. The conclusion of the corollary now implies the independent set theorem.

The remainder of the paper is organized as follows: In Section 2 we reduce the main theorem to a particular class of maps $\varphi$ related to matching monoids of complete bipartite graphs. For these maps, finite generation of the kernel follows from recent results on the Birkhoff model [Yamaguchi et al. 2014]; see Section 3, where we also describe the image of $\varphi$. In Section 4 we prove Noetherianity of $\operatorname{im} \varphi$, still for our special $\varphi$. As in [Cohen 1987; Hillar and Sullivant 2012], the strategy in Section 4 is to prove that a partial order on certain monoids is a wellpartial-order. In our case, these are said to be matching monoids, and the proof that they are well-partially ordered is quite subtle. In Section 5 we establish that a finite $\operatorname{Inc}(\mathbb{N})$-generating set of $\operatorname{ker} \varphi$ is (at least theoretically) computable. The last section describes a simpler procedure that one can attempt in order to obtain a generating set; at the moment, we do not know if this procedure is guaranteed to terminate. We conclude the paper with a computational example for which termination does occur.

\section{Reduction to matching monoids}

In this section we reduce the main theorem to a special case to be treated in the next two sections. To formulate this special case, let $N \in \mathbb{N}$ and for each $p \in[N]$ let $k_{p} \in \mathbb{N}$. First, introduce a set $Y^{\prime}$ of variables $y_{p, J}^{\prime}$ where $p \in[N]$ and $J=\left(j_{l}\right)_{l \in\left[k_{p}\right]} \in \mathbb{N}^{\left[k_{p}\right]}$ is a $k_{p}$-tuple of distinct natural numbers. The group $\operatorname{Sym}(\mathbb{N})$ acts on $Y^{\prime}$ by $\pi y_{p, J}^{\prime}=y_{p, \pi(J)}^{\prime}$ where $\pi(J)=\left(\pi\left(j_{l}\right)\right)_{l \in\left[k_{p}\right]}$. This action has finitely many orbits and satisfies the condition preceding the main theorem. 
Second, let $X$ be a set of variables $x_{p, l, j}$ with $p \in[N], l \in\left[k_{p}\right], j \in \mathbb{N}$ and let $\operatorname{Sym}(\mathbb{N})$ act on $X$ by its action on the last index.

Proposition 2.1. Let $\varphi^{\prime}: R\left[Y^{\prime}\right] \rightarrow R[X]$ be the R-algebra homomorphism sending $y_{p, J}^{\prime}$ to $\prod_{l \in\left[k_{p}\right]} x_{p, l, j_{l}}$. Then the main theorem implies that $\operatorname{ker} \varphi^{\prime}$ is generated by finitely many $\operatorname{Inc}(\mathbb{N})$-orbits of binomials, and that $\operatorname{im} \varphi^{\prime}$ is an $\operatorname{Inc}(\mathbb{N})$-Noetherian ring. Conversely, if these two statements hold for all choices of $N, k_{1}, \ldots, k_{N} \in \mathbb{N}$, then the main theorem holds.

Proof. The first statement is immediate - note that the pair $(p, l)$ comprising the first two indices of the variables $x_{p, l, j}$ takes on finitely many, namely $\sum_{p} k_{p}$, values.

For the second statement, consider a monomial map $\varphi: R[Y] \rightarrow R[Z]$ with $Z=\left\{z_{i, j} \mid i \in[k], j \in \mathbb{N}\right\}$ as in the main theorem. Let $N$ be the number of $\operatorname{Sym}(\mathbb{N})$ orbits on $Y$ and let $y_{p}, p \in[N]$ be representatives of the orbits. Set $k_{p}:=k_{y_{p}}$ for $p \in[N]$, so that $\pi y_{p}$ depends only on the restriction of $\pi \in \operatorname{Sym}(\mathbb{N})$ to $\left[k_{p}\right]$. We have thus determined the values of $N$ and the $k_{p}$, and we let $Y^{\prime}, X$ be as above.

Let $\psi: R\left[Y^{\prime}\right] \rightarrow R[Y]$ be the $R$-algebra homomorphism defined by sending $y_{p, J}^{\prime}$ to $\pi y_{p}$ for any $\pi \in \operatorname{Sym}(\mathbb{N})$ satisfying $\pi(l)=j_{l}, l \in\left[k_{p}\right]$. This homomorphism is $\operatorname{Sym}(\mathbb{N})$-equivariant. The composition $\varphi^{\prime \prime}:=\varphi \circ \psi: R\left[Y^{\prime}\right] \rightarrow R[Z]$ satisfies the conditions of the main theorem. Since $\psi$ is surjective, it maps any generating set for $\operatorname{ker} \varphi^{\prime \prime}$ onto a generating set for $\operatorname{ker} \varphi$; moreover, we have $\operatorname{im} \varphi^{\prime \prime}=\operatorname{im} \varphi$. Hence the conclusions of the main theorem for $\varphi^{\prime \prime}$ imply those for $\varphi$.

Next write $\varphi^{\prime \prime}\left(y_{p, J}\right)=\prod_{i \in[k], j \in \mathbb{N}} z_{i, j}^{d_{p, i, j}}$. Observe that $d_{p, i, j}=0$ whenever $j \notin J$, using the fact that any permutation that fixes $J$ also fixes $y_{p, J}$, and hence must also fix $\varphi^{\prime \prime}\left(y_{p, J}\right)$ by $\operatorname{Sym}(\mathbb{N})$-equivariance. Now let $\varphi^{\prime}: K\left[Y^{\prime}\right] \rightarrow K[X]$ be as above and define $\rho: R[X] \rightarrow R[Z]$ by $\rho\left(x_{p, l, j}\right)=\prod_{i \in[k]} z_{i, j}^{d_{p, i, j}}$. By construction, we have $\rho \circ \varphi^{\prime}=\varphi^{\prime \prime}$.

Now $\operatorname{im} \varphi^{\prime \prime}$ is a quotient of $\operatorname{im} \varphi^{\prime}$ and $\operatorname{ker} \varphi^{\prime \prime}$ is generated by $\operatorname{ker} \varphi^{\prime}$ together with preimages of generators of $\operatorname{ker}\left(\left.\rho\right|_{\mathrm{im}} \varphi^{\prime}\right)$, hence the conclusions of the main theorem for $\varphi^{\prime}$ imply those for $\varphi^{\prime \prime}$, as desired.

In what follows, we will drop the accents on the $y$-variables and write $Y$ for the set of variables $y_{p, J}, X$ for the set of variables $x_{p, l, j}$, and $\varphi$ for the $R$-algebra homomorphism

$$
\varphi: R[Y] \rightarrow R[X], y_{p, J} \mapsto \prod_{l \in\left[k_{p}\right]} x_{p, l, j_{l}} .
$$

Monomials in the $x_{p, l, j}$ will be denoted $x^{A}$ where $A \in \prod_{p \in[N]} \mathbb{N}^{\left[k_{p}\right] \times \mathbb{N}}$ is an $[N]$ tuple of finite-by-infinite matrices $A_{p}$. Note that $\varphi\left(y_{p, J}\right)$ equals $x^{A}$ where only the $p$-th component $A_{p}$ of $A$ is nonzero and in fact has all row sums equal to 1 , all column sums labeled by $J$ equal to 1 , and all other column sums equal to 0 . Thus $A_{p}$ can be thought of as the adjacency matrix of a matching of the maximal size $k_{p}$ 
in the complete bipartite graph with bipartition $\left[k_{p}\right] \sqcup \mathbb{N}$. Thus the monomials in $\operatorname{im} \varphi$ form the abelian monoid generated by such matchings (with $p$ varying). We call a monoid like this a matching monoid. In the next section we characterize these monomials among all monomials in the $x_{p, l, j}$, and find a bound on the relations among the $\varphi\left(y_{p, J}\right)$.

\section{Relations among matchings}

We retain the setting at the end of the previous section: $Y$ is the set of variables $y_{p, J}$ with $p$ running through $[N]$ and $J \in \mathbb{N}^{\left[k_{p}\right]}$ running through the $\left[k_{p}\right]$-tuples of distinct natural numbers; $X$ is the set of variables $x_{p, l, j}$ with $p \in[N], l \in\left[k_{p}\right], j \in \mathbb{N}$, and $\varphi$ is the map in (1). In this section we describe both the kernel and the image of $\varphi$. Note that if some $k_{p}$ is zero, then the corresponding (single) variable $y_{p,()}$ is mapped by $\varphi$ to 1 . The image of $\varphi$ does not change if we disregard those $p$, and the kernel changes only in that we forget about the generators $y_{p,()}-1$. Hence we may and will assume that all $k_{p}$ are strictly positive. The following lemma gives a complete characterization of the $x^{A}$ in the image of $\varphi$.

Proposition 3.1. For an $[N]$-tuple $A \in \prod_{p \in[N]} \mathbb{N}^{\left[k_{p}\right] \times \mathbb{N}}$ the monomial $x^{A}$ lies in the image of $\varphi$ if and only if for all $p \in[N]$ the matrix $A_{p} \in \mathbb{N}^{\left[k_{p}\right] \times \mathbb{N}}$ has all row sums equal to a number $d_{p} \in \mathbb{N}$ and all column sums less than or equal to $d_{p}$.

We call such $A$ good. Note that $d_{p}$ is unique since all $k_{p}$ are strictly positive. We call the vector $\left(d_{p}\right)_{p}$ the multidegree of $A$ and of $x^{A}$.

Remark 3.2. By replacing $\mathbb{N}$ with $[n]$ for some natural number $n$ greater than or equal to the maximum of the $k_{p}$, the proposition boils down to the statement that for each $p$ the lattice polytope in $\mathbb{R}^{\left[k_{p}\right] \times[n]}$ with defining inequalities $\forall_{i j} a_{i j} \geq 0$, $\forall_{i} \sum_{j} a_{i j}=1$, and $\forall_{j} \sum_{i} a_{i j} \leq 1$ is normal (in the case where $n=k_{p}$ this is the celebrated Birkhoff polytope). This is a not new result; in fact, this polytope satisfies a stronger property, namely, it is compressed. This follows, for instance, from [Sullivant 2006, Theorem 2.4] or from the main theorem of [Ohsugi and Hibi 2001]; see also [Yamaguchi et al. 2014, Section 4.2]. For completeness, we include a proof of the proposition using elementary properties of matchings in bipartite graphs.

Proof of Proposition 3.1. Let $x_{p}$ denote the vector of variables $x_{p, l, j}$ for $l \in\left[k_{p}\right]$ and $j \in \mathbb{N}$. By definition of $\varphi$, the monomial $x^{A}$ lies in $\operatorname{im} \varphi$ if and only if the monomial $x_{p}^{A_{p}}$ lies in $\operatorname{im} \varphi$ for all $p \in[N]$. Thus it suffices to prove that $x_{p}^{A_{p}}$ lies in $\operatorname{im} \varphi$ if and only if all row sums of $A_{p}$ are equal, say to $d \in \mathbb{N}$, and all column sums of $A_{p}$ are at most $d$. The "only if" part is clear, since every variable $y_{p, J}$ is mapped to a monomial $x_{p}^{B}$ where $B \in \mathbb{N}^{\left[k_{p}\right] \times \mathbb{N}}$ has all row sums 1 and all column sums at most 1 . For the "if" part we proceed by induction on $d$ : assume that the 


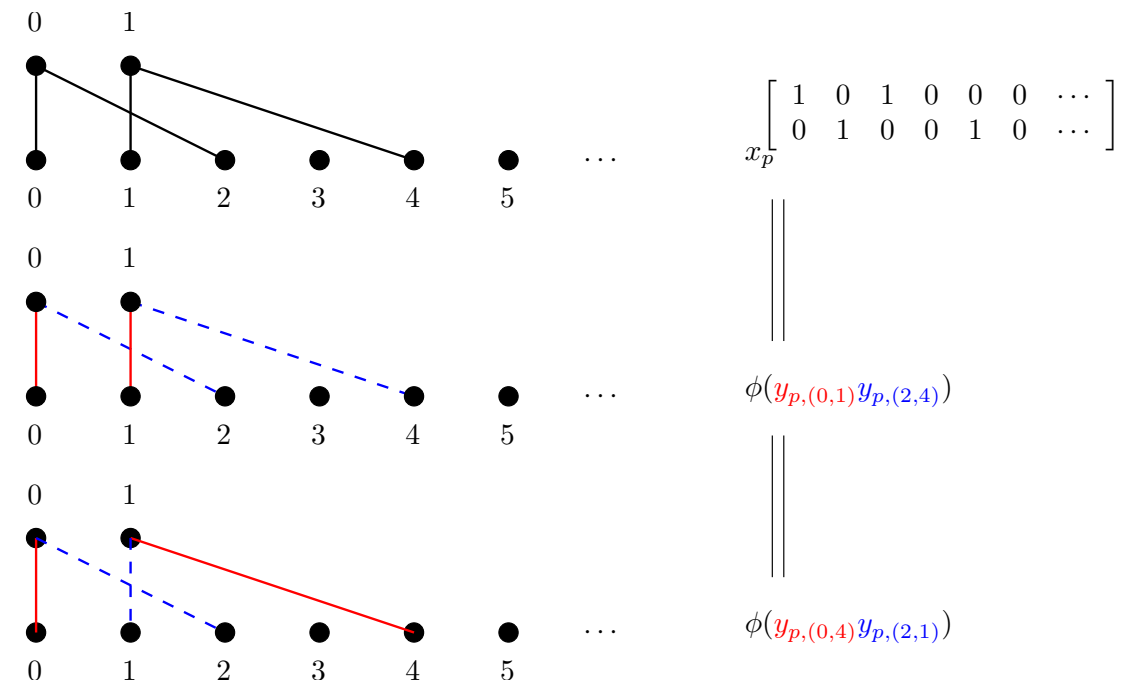

Figure 1. A bipartite graph on [2] $\sqcup \mathbb{N}$ and its corresponding monomial $x_{p}^{A_{p}}$ (top). This graph can be decomposed into matchings in two different ways (middle and bottom). Each decomposition represents a monomial in the preimage $\varphi^{-1}\left(x_{p}^{A_{p}}\right)$.

statement holds for $d-1$, and consider a matrix $A_{p}$ with row sums $d$ and column sums $\leq d$, where $d$ is at least 1. Clearly, the "if" part is true in the case $d=0$.

Think of $A_{p}$ as the adjacency matrix of a bipartite graph $\Gamma$ (with multiple edges) with bipartition $\left[k_{p}\right] \sqcup \mathbb{N}$ (see Figure 1). With this viewpoint in mind, we will invoke some standard results from combinatorics, and refer to [Schrijver 2003, Chapter 16]. The first observation is that $\Gamma$ contains a matching that covers all vertices in $\left[k_{p}\right]$. Indeed, otherwise, by Hall's marriage theorem, after permuting rows and columns, $A_{p}$ has the block structure

$$
A_{p}=\left[\begin{array}{cc}
A_{11} & 0 \\
A_{12} & A_{22}
\end{array}\right]
$$

with $A_{11} \in \mathbb{N}^{[l] \times[l-1]}$ for some $l$ satisfying $1 \leq l \leq k_{p}$. But then the entries of $A_{11}$ added row-wise add up to $l d$, and added columnwise add up to at most $(l-1) d$, a contradiction. Hence $\Gamma$ contains a matching that covers all of $\left[k_{p}\right]$. Next, let $S \subseteq \mathbb{N}$ be the set of column indices where $A_{p}$ has column sum equal to the upper bound $d$. We claim that $\Gamma$ contains a matching that covers all of $S$. Indeed, otherwise, again by Hall's theorem, after permuting rows and columns $A_{p}$ has the structure

$$
A_{p}=\left[\begin{array}{cc}
A_{11} & A_{12} \\
0 & A_{22}
\end{array}\right]
$$


with $A_{11} \in \mathbb{N}^{[l-1] \times[l]}$ for some $l$ with $1 \leq l \leq|S|$; here the first $l$ columns correspond to a subset of the original $S$. Now the entries of $A_{11}$ added columnwise yield $l d$, while the entries of $A_{11}$ added row-wise yield at most $(l-1) d$, a contradiction.

Finally, we invoke a standard result in matching theory (see [Schrijver 2003, Theorem 16.8]), namely that since $\Gamma$ contains a matching that covers all of $\left[k_{p}\right]$ and a matching that covers all of $S$, it also contains a matching that covers both. Let $B$ be the adjacency matrix of this matching, so that $B$ has all row sums 1 and all column sums $\leq 1$, with equality at least in the columns labeled by $S$. Then $A_{p}^{\prime}:=A_{p}-B$ satisfies the induction hypothesis for $d-1$, so $x_{p}^{A_{p}^{\prime}} \in \operatorname{im} \varphi$. Also, $x_{p}^{B}=\varphi\left(y_{p, J}\right)$, where $j_{a} \in \mathbb{N}$ is the neighbor of $a \in\left[k_{p}\right]$ in the matching given by $B$. Hence, $x_{p}^{A_{p}}=x_{p}^{A_{p}^{\prime}} x_{p}^{B} \in \operatorname{im} \varphi$ as claimed.

This concludes the description of the image of $\varphi$. For the kernel, we quote the following result.

Theorem 3.3 [Yamaguchi et al. 2014, Theorem 2.1]. The kernel of $\varphi$ from (1) is generated by binomials in the $y_{p, J}$ of degree at most 3.

Indeed, for each fixed $p$, and replacing $\mathbb{N}$ by some $[n]$ with $n \geq k_{p}$, the monomial map (1) captures precisely the generalization of the Birkhoff model studied in [Yamaguchi et al. 2014], where each voter chooses $k_{p}$ among $n$ candidates. Then their Theorem 2.1 yields that the kernel is generated in degrees 2 and 3. Since this holds for each $n \geq k_{p}$, it also holds for $\mathbb{N}$ instead of $[n]$. Moreover, taking the union over all $p$ of sets of generators for each individual $p$ yields a set of generators for the kernel of $\varphi$. A straightforward consequence of the theorem is the following.

Corollary 3.4. The kernel of $\varphi$ from (1) is generated by finitely many $\operatorname{Inc}(\mathbb{N})$-orbits of binomials.

\section{Noetherianity of matching monoid rings}

By Corollary 3.4 and Proposition 2.1, the main theorem follows from the following proposition.

Proposition 4.1. The ring $R\left[x^{A} \mid A \in \prod_{p \in[N]} \mathbb{N}^{\left[k_{p}\right] \times \mathbb{N}}\right.$ good $]$ is $\operatorname{Inc}(\mathbb{N})$-Noetherian.

Let $S$ be the ring in the proposition, and let $G \subset \prod_{p \in[N]} \mathbb{N}^{\left[k_{p}\right] \times \mathbb{N}}$ be the set of $\operatorname{good}\left(N\right.$-tuples of) matrices. The monomials of $S$ are precisely $x^{A}$, for $A \in G$. The monoids $\operatorname{Sym}(\mathbb{N})$ and $\operatorname{Inc}(\mathbb{N})$ act on $G$ by permuting or shifting columns, so we have $\pi x^{A}=x^{\pi A}$, where the $\pi(j)$-th column of the matrix $(\pi A)_{p}$ equals the $j$-th column of $A_{p}$. Let $d_{A}=\left(d_{A, p}\right)_{p} \in \mathbb{N}^{[N]}$ denote the multidegree of $A$; recall that this means that all row sums of $A_{p}$ are equal to $d_{A, p}$. To prove Noetherianity we will define a partial order $\preceq$ on $G$ and prove that $\preceq$ is a well-partial-order. Thus we need some basic results from order theory. 
A partial order $\preceq$ on a set $P$ is a well-partial-order (or wpo) if for every infinite sequence $p_{1}, p_{2}, \ldots$ in $P$, there is some $i<j$ such that $p_{i} \preceq p_{j}$; see [Kruskal 1972] for alternative characterizations. For instance, the natural numbers with the usual total order $\leq$ is a well-partial-order, and so is the componentwise partial order on the Cartesian product of any finite number of well-partially ordered sets. Combining these statements yields Dickson's lemma [1913] that $\mathbb{N}^{k}$ is well-partially ordered. This can be seen as a special case of Higman's lemma [1952], for a beautiful proof of which we refer to [Nash-Williams 1963].

Lemma 4.2 (Higman's lemma). Let $(P, \preceq)$ be a well-partial-order and let $P^{*}:=$ $\bigcup_{l=0}^{\infty} P^{l}$, the set of all finite sequences of elements of $P$. Define the partial order $\preceq^{\prime}$ on $P^{*}$ by $\left(a_{0}, \ldots, a_{l-1}\right) \preceq^{\prime}\left(b_{0}, \ldots, b_{m-1}\right)$ if and only if there exists a strictly increasing function $\rho:[l] \rightarrow[m]$ such that $a_{j} \preceq b_{\rho(j)}$ for all $j \in[l]$. Then $\preceq^{\prime}$ is a well-partial-order.

Our interest in well-partial-orders stems from the following application. Consider a commutative monoid $\mathrm{M}$ with an action of a (typically noncommutative) monoid $\Pi$ by means of monoid endomorphisms. We suggestively call the elements of $\mathrm{M}$ monomials. Assume that we have a П-compatible monomial order $\leq$ on $\mathrm{M}$, i.e., a well-order that satisfies $a<b \Rightarrow a c<b c$ and $a<b \Rightarrow \pi a<\pi b$ for all $a, b, c \in \mathrm{M}$ and $\pi \in \Pi$. Then it follows that the divisibility relation $\mid$ defined by $a \mid b$ if there exists a $c \in \mathrm{M}$ with $a c=b$ is a partial order, and also that $a \leq \pi a$ for all $a \in \mathrm{M}$. Define a third partial order, the П-divisibility order, $\preceq$ on $\mathrm{M}$ by $a \preceq b$ if there exists a $\pi \in \Pi$ and a $c \in \mathrm{M}$ such that $c \pi a=b$. A straightforward computation shows that $\preceq$ is, indeed, a partial order - antisymmetry follows using $a \leq \pi a$.

Proposition 4.3. If $\preceq$ is a well-partial-order, then for any Noetherian ring $R$, the $R$-algebra $R[\mathrm{M}]$ is $\Pi$-Noetherian.

Proof. This statement was proved in [Hillar and Sullivant 2012] for the case where $R$ is a field. The more general case can be proved with the same argument by incorporating work done in [Aschenbrenner and Hillar 2007].

Note that the monoid $\left\{x^{A} \mid A \in G\right\}$ that we are considering here can be given a monomial order which respects the $\operatorname{Inc}(\mathbb{N})$-action. For example, take the lexicographic order, where the variables $x_{p, i, j}$ are ordered by their indices: $x_{p, i, j}<x_{p^{\prime}, i^{\prime}, j^{\prime}}$ if and only if $p<p^{\prime}$; or $p=p^{\prime}$ and $j<j^{\prime}$; or $p=p^{\prime}, j=j^{\prime}$, and $i<i^{\prime}$.

The $\operatorname{Inc}(\mathbb{N})$-divisibility order gives a partial order $\preceq$ on the set $G$ of $\operatorname{good}(N$ tuples of) matrices by $A \preceq B$ if and only if there is a monomial $x^{C} \in S$ and $\pi \in \operatorname{Inc}(\mathbb{N})$ such that $x^{C} \pi\left(x^{A}\right)=x^{B}$, or equivalently there is $\pi \in \operatorname{Inc}(\mathbb{N})$ such that $B-\pi A \in G$. Note that $A \preceq B$ not only implies there is some $\pi \in \operatorname{Inc}(\mathbb{N})$ such that all $A_{p, i, j} \leq B_{p, i, \pi(j)}$, but additionally that all ( $N$-tuples of) column sums of $B-\pi A$ are at most $d_{B}-d_{A} \in \mathbb{N}^{[N]}$. This prevents us from applying Higman's 
lemma directly to $(G, \preceq)$. To encode this condition on column sums, for any $A \in G$, let $\tilde{A} \in \prod_{p \in[N]} \mathbb{N}^{\left[k_{p}+1\right] \times \mathbb{N}}$ be the $N$-tuple of matrices such that for all $p \in[N]$, the first $k_{p}$ rows of $\tilde{A}_{p}$ are equal to $A_{p}$, and the last row of $\tilde{A}_{p}$ is such that all column sums equal $d_{A, p}$ :

$$
\tilde{A}_{p, i, j}= \begin{cases}A_{p, i, j} & \text { for } i<k_{p}, \\ d_{A, p}-\sum_{l=0}^{k_{p}-1} A_{p, l, j} & \text { for } i=k_{p} .\end{cases}
$$

We let $\tilde{G}$ be the set of $N$-tuples of matrices of the form $\tilde{A}$ with $A \in G$. It is precisely the set of $N$-tuples of matrices of the form $\tilde{A} \in \prod_{p \in[N]} \mathbb{N}^{\left[k_{p}+1\right] \times \mathbb{N}}$ with the property that there exists a $d_{A} \in \mathbb{N}^{[N]}$ such that for each $p \in[N]$ the first $k_{p}$ row sums of $A_{p}$ are equal to $d_{A, p}$ and all column sums of $A_{p}$ are equal to $d_{A, p}$. Since $A \in G$ has only finitely many $N$-tuples of nonzero columns, $\tilde{A}$ will have all but finitely many $N$-tuples of columns equal to $\left(\left(0, \ldots, 0, d_{A, p}\right)^{T}\right)_{p \in[N]}$. Such $N$-tuples of columns will be called trivial (of degree $d_{A}$ ). The $N$-tuple of $j$-th columns of $\tilde{A}$ will be denoted $\tilde{A}_{. . j}$. We define the action of $\operatorname{Inc}(\mathbb{N})$ on $\tilde{G}$ as $\pi(\tilde{A})=\widetilde{\pi(A)}$. Note that for any $j \notin \operatorname{im}(\pi)$, the column $(\pi \tilde{A})_{. . j}$ is trivial of degree $d_{A}$, rather than uniformly zero.

Proposition 4.4. For $A, B \in G, A \preceq B$ if and only if there is $\pi \in \operatorname{Inc}(\mathbb{N})$ such that $\pi \tilde{A} \leq \tilde{B}$ entrywise.

Proof. The condition that $(\pi \tilde{A})_{p, i, j} \leq \tilde{B}_{p, i, j}$ for all $p \in[N]$, all $i<k_{p}$, and all $j \in \mathbb{N}$ is equivalent to the condition that $B-\pi A$ is nonnegative. Using the fact that

$$
\tilde{B}_{p, k_{p}, j}-(\pi \tilde{A})_{p, k_{p}, j}=\left(d_{B, p}-d_{A, p}\right)-\sum_{i=0}^{k_{p}-1}\left(B_{p}-\pi A_{p}\right)_{i, j},
$$

the condition that $\tilde{B}_{p, k_{p}, j}-(\pi \tilde{A})_{p, k_{p}, j} \geq 0$ for all $p \in[N]$ and all $j \in \mathbb{N}$ is equivalent to the condition that every $N$-tuple of column sums of $B-\pi A$ is less than or equal to $d_{B}-d_{A}$. Therefore $\pi \tilde{A} \leq \tilde{B}$ if and only if $B-\pi A \in G$.

Example 4.5. Let $A$ and $B$ be the following good matrices in $\mathbb{N}^{[2] \times \mathbb{N}}$ :

$$
A=\left[\begin{array}{llllll}
3 & 0 & 0 & 0 & 0 & \cdots \\
0 & 1 & 1 & 1 & 0 & \cdots
\end{array}\right], \quad B=\left[\begin{array}{llllll}
3 & 1 & 0 & 0 & 0 & \cdots \\
0 & 2 & 1 & 1 & 0 & \cdots
\end{array}\right]
$$

Note that $\pi A \leq B$ when $\pi$ is the identity, however $A \npreceq B$. Consider

$$
\tilde{A}=\left[\begin{array}{llllll}
3 & 0 & 0 & 0 & 0 & \cdots \\
0 & 1 & 1 & 1 & 0 & \cdots \\
0 & 2 & 2 & 2 & 3 & \cdots
\end{array}\right], \quad \tilde{B}=\left[\begin{array}{llllll}
3 & 1 & 0 & 0 & 0 & \cdots \\
0 & 2 & 1 & 1 & 0 & \cdots \\
1 & 1 & 3 & 3 & 4 & \cdots
\end{array}\right],
$$

and note that there is no $\pi \in \operatorname{Inc}(\mathbb{N})$ such that $\pi \tilde{A} \leq \tilde{B}$. 
We will work with finite truncations of $N$-tuples of matrices in $\tilde{G}$. Let $\mathrm{H}$ be the set of $N$-tuples of matrices $A \in \bigcup_{\ell=0}^{\infty} \prod_{p \in[N]} \mathbb{N}^{\left[k_{p}+1\right] \times[\ell]}$ such that there exists $d_{A} \in \mathbb{N}^{[N]}$ such that for all $p$, all column sums of $A_{p}$ are equal to $d_{A, p}$ and the first $k_{p}$ row sums are at most $d_{A, p}$; we call $d_{A}$ the multidegree of $A$. Note that the condition on row sums is relaxed, which will allow us to freely remove columns from matrices while still remaining in the set $\mathrm{H}$. For $A \in \mathrm{H}$ the number of columns of $A$ is called the length of $A$ and denoted $\ell_{A}$. We give $H$ the partial order $\preceq$ defined as follows. For $A, B \in \mathrm{H}, A \preceq B$ if and only if there is a strictly increasing map $\rho:\left[\ell_{A}\right] \rightarrow\left[\ell_{B}\right]$ such that $\rho A \leq B$. Just as in $\tilde{G}$, here $\rho A$ is defined by $(\rho A)_{. . j}=A_{\ldots \rho^{-1}(j)}$ for $j \in \operatorname{im}(\rho)$, and $(\rho A)_{. . j}$ trivial (of degree $d_{A}$ ) for $j \in\left[\ell_{B}\right] \backslash \operatorname{im}(\rho)$. For an $N$-tuple of matrices $A$ and a set $J \subset \mathbb{N}$, let $A$... denote the $N$-tuple of matrices obtained from $A$ by taking only the columns $A . . j$ with $j \in J$.

Some care must be taken in the definition of $\mathrm{H}$ since we allow matrices with no columns. In all other cases, the degree of $A \in \mathrm{H}$ is uniquely determined by its entries. However for the length 0 case the degree is arbitrary, so we will consider $\mathrm{H}$ as having a distinct length 0 element $Z^{d}$ with degree $d$ for each $d \in \mathbb{N}^{[N]}$, and we define $Z^{d} \preceq A$ if and only if $d \leq d_{A}$. Additionally, define $A . . \varnothing=Z^{d_{A}}$.

Definition 4.6. For $A \in \mathrm{H}$, the $N$-tuple of $j$-th columns of $A$ is bad if for some $p \in[N]$, we have $A_{p, k_{p}, j}<d_{A, p} / 2$. If $A_{p, k_{p}, j}<d_{A, p} / 2$, we will call $j$ a bad index of $A$ (with respect to $p$ ). Let $\mathrm{H}_{t}$ denote the set of $N$-tuples of matrices in $\mathrm{H}$ with exactly $t$ bad indices.

We will use induction on $t$ to show that $\left(\mathrm{H}_{t}, \preceq\right)$ is well-partially ordered for all $t \in \mathbb{N}$. This will in turn be used to prove that $(\mathrm{H}, \preceq)$ and then $(\tilde{G}, \preceq)$ are well-partially ordered. First we prove the base case:

Proposition 4.7. $\left(\mathrm{H}_{0}, \preceq\right)$ is well-partially ordered.

Proof. Let $A^{(1)}, A^{(2)}, \ldots$ be any infinite sequence in $\mathrm{H}_{0}$. We will show that there is an $r$ and an $s$, with $r<s$, such that $A^{(r)} \preceq A^{(s)}$.

Fix $p \in[N]$. There are now two possibilities: either the degrees of the elements of the sequence $A_{p}^{(1)}, A_{p}^{(2)}, \ldots$ are bounded by some $d_{p} \in \mathbb{N}$, or they are not. In the former case, it follows that the number of nontrivial columns in any $A_{p}^{(r)}$ is bounded by $d_{p} k_{p}$. Then there is a subsequence $B_{p}^{(1)}, B_{p}^{(2)}, \ldots$ of $A_{p}^{(1)}, A_{p}^{(2)}, \ldots$ such that every element has the same degree and same number of nontrivial columns. In the latter case, $A_{p}^{(1)}, A_{p}^{(2)}, \ldots$ has a subsequence with strictly increasing degree and moreover a subsequence $B_{p}^{(1)}, B_{p}^{(2)}, \ldots$ with the property that $d_{B^{(s+1)}, p} \geq 2 d_{B^{(s)}, p}$ for all $s \in \mathbb{N}$.

In either case we replace $A^{(1)}, A^{(2)}, \ldots$ by $B^{(1)}, B^{(2)}, \ldots$ without loss of generality. We repeat this procedure for all $p \in[N]$, and we find that $A^{(1)}, A^{(2)}, \ldots$ contains a subsequence $B^{(1)}, B^{(2)}, \ldots$ such that for all $p \in[N]$, one of the following two statements holds. 
1: Both $d_{B^{(t)}, p}$ and the number of nontrivial columns in $B_{p}$ are constant.

2: We have $d_{B^{(t+1)}, p} \geq 2 d_{B^{(t)}, p}$ for all $t$.

It now suffices to show that there are $r<s$ such that $B^{(r)} \preceq B^{(s)}$. Define the partial order $\sqsubseteq$ on $\mathrm{H}_{0}$ by $A \sqsubseteq B$ if and only if there exists strictly increasing $\rho:\left[\ell_{A}\right] \rightarrow\left[\ell_{B}\right]$ such that $A . . j \leq B . . \rho(j)$ for all $j \in\left[\ell_{A}\right]$. By Higman's lemma (Lemma 4.2), $\sqsubseteq$ is a wpo. This means that there exist $r<s$ such that $B^{(r)} \sqsubseteq B^{(s)}$. Fix such a pair $r<s$. We will show that $B^{(r)} \preceq B^{(s)}$.

Let $\rho:\left[\ell_{B^{(r)}}\right] \rightarrow\left[\ell_{B^{(s)}}\right]$ be a strictly increasing map that witnesses $B^{(r)} \sqsubseteq B^{(s)}$. We claim that it also witnesses $B^{(r)} \preceq B^{(s)}$. For this, we have to show that $\rho B^{(r)} \leq B^{(s)}$. By the properties of $\sqsubseteq$, we already have $\left(\rho B^{(r)}\right)_{. . \rho(j)} \leq B_{. . \rho(j)}^{(s)}$, which is to say that it suffices to show that for all $j \notin \operatorname{im}(\rho)$, we have $d_{B^{(r)}} \leq\left(B_{p, k_{p}, j}^{(s)}\right)_{p \in[N]}$.

Let $p \in[N]$. Suppose we are in the case that both $d_{B^{(t)}, p}$ and the number of nontrivial columns in $B_{p}$ are constant. Since $\rho$ must map nontrivial columns of $B_{p}^{(r)}$ to nontrivial columns of $B_{p}^{(s)}$, we conclude that if $j \notin \operatorname{im}(\rho)$, then the $j$-th column of $B_{p}^{(s)}$ is trivial, and hence $\left(B_{p, k_{p}, j}^{(s)}\right)=d_{B^{(s)}, p}$. But the latter equals $d_{B^{(r)}, p}$, so certainly $d_{B^{(r)}, p} \leq\left(B_{p, k_{p}, j}^{(s)}\right)$.

Alternatively, suppose we have $d_{B^{(t+1)}, p} \geq 2 d_{B^{(t)}, p}$ for all $t$. Since $B_{p}^{(s)}$ has no bad columns, we have

$$
B_{p, k_{p}, j}^{(s)} \geq \frac{1}{2} d_{B^{(s)}, p} \geq d_{B^{(r)}, p} .
$$

This is exactly what we wanted to show.

So in both cases, we find that $d_{B^{(r)}, p} \leq B_{p, k_{p}, j}^{(s)}$ for all $j \notin \operatorname{im}(\rho)$. This is true for all $p$, so we have $d_{B^{(r)}} \leq\left(B_{p, k_{p}, j}^{(s)}\right)_{p \in[N]}$. We conclude that $B^{(r)} \preceq B^{(s)}$, as we wanted to show.

Proposition 4.8. $\left(\mathrm{H}_{t}, \preceq\right)$ is well-partially ordered for all $t \in \mathbb{N}$.

Proof. The base case, $t=0$, is given by Proposition 4.7. For $t>0$, assume by induction that $\left(\mathrm{H}_{t-1}, \preceq\right)$ is well-partially ordered. For any $A \in \mathrm{H}_{t}$, let $j_{A}$ be the largest bad index of $A$. Then $A$ can be decomposed into three parts: the $N$-tuple of matrices of all $N$-tuples of columns before $j_{A}, A_{. . j_{A}}$ itself, and the $N$-tuple of matrices of all $N$-tuples of columns after $j_{A}$. This decomposition is represented by the map

$$
\begin{aligned}
\delta: \mathrm{H}_{t} & \rightarrow \mathrm{H}_{t-1} \times \prod_{p \in[N]} \mathbb{N}^{\left[k_{p}+1\right]} \times \mathrm{H}_{0} \\
A & \mapsto\left(A . .\left\{0, \ldots, j_{A}-1\right\}, A . . j_{A}, A . .\left\{j_{A}+1, \ldots, \ell_{A}-1\right\}\right) .
\end{aligned}
$$

Let the partial order $\sqsubseteq$ on $\mathrm{H}_{t-1} \times \prod_{p \in[N]} \mathbb{N}^{\left[k_{p}+1\right]} \times \mathrm{H}_{0}$ be the product order of the wpos $\left(\mathrm{H}_{t-1}, \preceq\right),\left(\mathbb{N}^{[k+1]}, \leq\right)$ and $\left(\mathrm{H}_{0}, \preceq\right)$. Note that the product order of any finite number of wpos is also a wpo. Suppose for some $A, B \in \mathrm{H}_{t}$ that $\delta(A) \sqsubseteq \delta(B)$. This implies that $A_{. . j_{A}} \leq B_{. . j_{B}}$ and that there exist strictly increasing maps $\rho$ and $\sigma$ such 


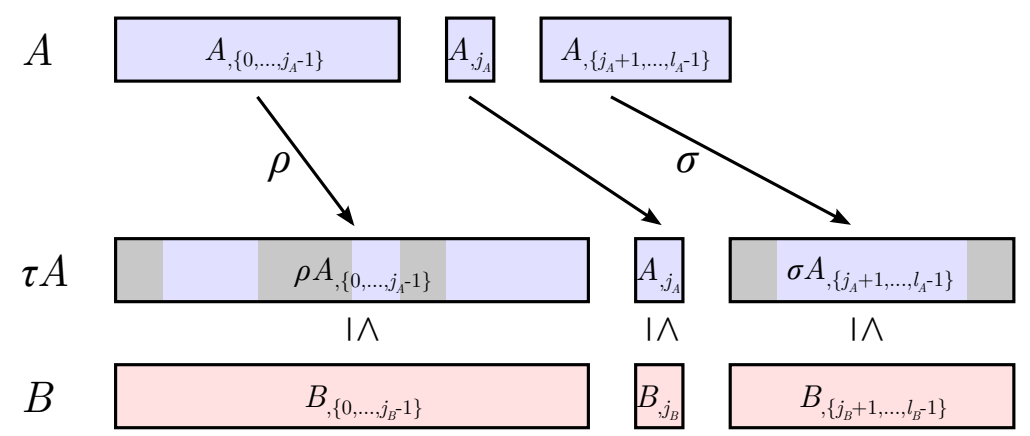

Figure 2. $\delta(A) \sqsubseteq \delta(B)$ implies $A \preceq B$.

that $\rho\left(A_{. .\left[j_{A}\right]}\right) \leq B_{. .\left[j_{B}\right]}$ and $\sigma\left(A . .\left\{j_{A}+1, \ldots, \ell_{A}-1\right\}\right) \leq B . .\left\{j_{B}+1, \ldots, \ell_{B}-1\right\}$. We combine these into a single strictly increasing map $\tau:\left[\ell_{A}\right] \rightarrow\left[\ell_{B}\right]$ defined by

$$
\tau(j)= \begin{cases}\rho(j) & \text { for } 0 \leq j<j_{A}, \\ j_{B} & \text { for } j=j_{A}, \\ \sigma\left(j-j_{A}-1\right)+j_{B}+1 & \text { for } j_{A}<j<\ell_{A},\end{cases}
$$

illustrated in Figure 2. Then $\tau A \leq B$ so $A \preceq B$. Since $\sqsubseteq$ is a wpo, $\left(\mathrm{H}_{t}, \preceq\right)$ is also a wpo.

Proposition 4.9. $(\mathrm{H}, \preceq)$ is well-partially ordered.

Proof. For any $A \in \mathrm{H}$, if $j$ is a bad index of $A$, then for some $p \in[N]$, we have $d_{A, p} / 2>\sum_{i \in\left[k_{p}\right]} A_{p, i, j}$. Letting $J_{p} \subset \mathbb{N}$ be the set of bad indices of $A$ with respect to $p$ and let $J \subset \mathbb{N}$ be the union of the $J_{p}$. Then

$$
\left|J_{p}\right| \frac{d_{A, p}}{2}<\sum_{j \in J_{p}} \sum_{i \in\left[k_{p}\right]} A_{p, i, j} \leq \sum_{i \in\left[k_{p}\right]} \sum_{j \in \mathbb{N}} A_{p, i, j} \leq k_{p} d_{A},
$$

with the last inequality due to the row sum condition on $A_{p}$. Therefore $\left|J_{p}\right| \leq 2 k_{p}-1$, and hence $|J| \leq 2 \sum_{p \in[N]} k_{p}-N$.

Let $A^{(1)}, A^{(2)}, \ldots$ be any infinite sequence in $\mathrm{H}$. Since the numbers of bad $N$-tuples of columns of elements of $\mathrm{H}$ are bounded by $2 \sum_{p \in[N]} k_{p}-N$ there exists a subsequence which is contained in $\mathrm{H}_{t}$ for some $0 \leq t \leq 2 \sum_{p \in[N]} k_{p}-N$. By Proposition 4.8 there is $r<s$ with $A^{(r)} \preceq A^{(s)}$.

Proposition 4.10. $(G, \preceq)$ is well-partially ordered.

Proof. Let $A^{(1)}, A^{(2)}, \ldots$ be any infinite sequence in $G$. Each $A^{(r)}$ has some $j_{r}>0$ such that all $N$-tuples of columns $A_{. \cdot m}^{(r)}$ are zero for $m \geq j_{r}$. Consider the sequence $\tilde{A}_{\cdot \cdot\left[j_{1}\right]}^{(1)}, \tilde{A}_{\cdot \cdot\left[j_{2}\right]}^{(2)}, \ldots$ in $\mathrm{H}$ obtained by truncating each $\tilde{A}^{(r)}$ to the first $j_{r} N$-tuples of columns. By Proposition 4.9 there is some $r<s$ and $\rho:\left[j_{r}\right] \rightarrow\left[j_{s}\right]$ such that 
$\rho \tilde{A}_{. \cdot\left[j_{r}\right]}^{(r)} \leq \tilde{A}_{. \cdot\left[j_{s}\right]}^{(s)}$. Note that this implies $d_{A^{(r)}} \leq d_{A^{(s)}}$. Extend $\rho$ to some $\pi \in \operatorname{Inc}(\mathbb{N})$ so then

$$
\left(\pi \tilde{A}^{(r)}\right)_{. \cdot\left[j_{s}\right]}=\rho\left(\tilde{A}_{\cdot \cdot\left[j_{r}\right]}^{(r)}\right) \leq \tilde{A}_{\cdot \cdot\left[j_{s}\right]}^{(s)} .
$$

The remaining $N$-tuples of columns of $\pi \tilde{A}^{(r)}$ and $\tilde{A}^{(s)}$ are trivial, so $\pi \tilde{A}^{(r)} \leq \tilde{A}^{(s)}$ follows from the fact that $d_{A^{(r)}} \leq d_{A^{(s)}}$. Therefore $A^{(r)} \preceq A^{(s)}$ by Proposition 4.4 .

Now we can apply Proposition 4.3 to the monoid $\left\{x^{A} \mid A \in G\right\}$ which proves that the ring $R\left[x^{A} \mid A \in G\right]$ is $\operatorname{Inc}(\mathbb{N})$-Noetherian. This concludes the proof of Proposition 4.1.

\section{Buchberger's algorithm for matching monoid algebras}

Assume the general setting of Proposition 4.3: $M$ is a monoid with $\Pi$-action and $\Pi$ compatible monomial order $\leq$. For a polynomial $f$ and an ideal $I$ in $K[M]$, we can define $\operatorname{lm}(f), \operatorname{lc}(f), \operatorname{in}(I)$, division with remainder, and the concept of equivariant Gröbner basis from [Brouwer and Draisma 2011]; all relative to the monomial order $\leq$. We now derive a version of Buchberger's algorithm for computing such a Gröbner basis, under an additional assumption. For $a, b \in M$ we define the set of least common multiples

$$
\operatorname{lcm}(a, b)=\left\{l \in M: a|l, b| l \text { and }\left(a\left|l^{\prime}, b\right| l^{\prime}, l^{\prime} \mid l \Rightarrow l^{\prime}=l\right)\right\} .
$$

We require the following variant of conditions EGB3 and EGB4 from [Brouwer and Draisma 2011]:

EGB34. For all $f, g \in K[M]$, the set of triples in $M \times \Pi f \times \Pi g$ defined by

$$
T_{f, g}=\left\{\left(l^{\prime}, f^{\prime}, g^{\prime}\right) \mid f^{\prime} \in \Pi f, g^{\prime} \in \Pi g, l^{\prime} \in \operatorname{lcm}\left(\operatorname{lm}\left(f^{\prime}\right), \operatorname{lm}\left(g^{\prime}\right)\right)\right\},
$$

is a union of a finite number of $\Pi$-orbits:

$$
T_{f, g}=\bigcup_{i} \Pi\left(l_{i}, f_{i}, g_{i}\right), \quad i \in[r] .
$$

In particular, EGB34 implies that for all $a, b \in M$ and $\pi \in \Pi$, we have $\pi \operatorname{lcm}(a, b) \subseteq$ $\operatorname{lcm}(\pi a, \pi b)$. (This is what condition EGB3 of [loc. cit.] looks like when least common multiples are not unique.)

If EGB34 is fulfilled, then there is a unique inclusion-minimal finite set of orbit generators as above, which we denote

$$
O_{f, g}=\left\{\left(l_{i}, f_{i}, g_{i}\right) \mid i \in[r]\right\} .
$$

Indeed, suppose that $O$ and $O^{\prime}$ are both inclusion-minimal sets of orbit generators for $T_{f, g}$. For any triple $t \in O$, there are $t^{\prime} \in O^{\prime}, \pi \in \Pi$ such that $\pi t^{\prime}=t$, and 
similarly $t^{\prime \prime} \in O, \tau \in \Pi$ such that $\tau t^{\prime \prime}=t^{\prime}$. Now $t=\pi \tau t^{\prime \prime}$ and since $O$ is minimal, $t=t^{\prime \prime}$. But since $\Pi$ is compatible with a monomial order, $\pi \tau t^{\prime \prime}=t^{\prime \prime}$ implies that also the intermediate expression $t^{\prime}=\tau t^{\prime \prime}$ equals $t^{\prime \prime}$. Hence $O \subseteq O^{\prime}$ and equality holds by minimality of $O^{\prime}$.

Definition 5.1. For monic $f, g \in K[M]$ define the set of S-polynomials to be

$$
S_{f, g}=\left\{a f^{\prime}-b g^{\prime} \mid\left(l^{\prime}, f^{\prime}, g^{\prime}\right) \in O_{f, g} ; a, b \in M ; \text { and } a \operatorname{lm}\left(f^{\prime}\right)=b \operatorname{lm}\left(g^{\prime}\right)=l^{\prime}\right\} .
$$

Furthermore, define $\Pi$-reduction of a polynomial $f$ with respect to a set $G \subseteq K[M]$ as follows: while there exist $g \in G$ and $\pi \in \Pi$ with $\pi \operatorname{lm}(g) \mid \operatorname{lm}(f)$, replace $f$ by

$$
f^{\prime}:=f-\frac{\operatorname{lc}(f) \operatorname{lm}(f)}{\operatorname{lc}(g) \pi \operatorname{lm}(g)} \pi g
$$

and when no such $g$ and $\pi$ exist, return the remainder $f^{\prime}$.

One can generalize Gröbner theory to our equivariant setting for a monoid algebra satisfying EGB34. In particular, Buchberger's criterion holds, and the following procedure produces an equivariant Gröbner basis if it terminates.

\section{Algorithm 5.2. $G=\operatorname{BuChBERGER}(F)$}

Require: $F$ is a finite set of monic elements in $K[M]$, the algebra of a monoid $M$ equipped with a $\Pi$-action, satisfying the assumptions above and the condition EGB34.

Ensure: $G$ is an equivariant Gröbner basis of $\langle F\rangle$.

1: $\quad G \leftarrow F$

2: $\quad S \leftarrow \bigcup_{f, g \in G} S_{f, g}\left\{\right.$ in particular, compute $O_{f, g}$ needed in Definition 5.1 $\}$

3: $\quad$ while $S \neq \varnothing$ do

4: $\quad$ pick $f \in S$

5: $\quad S \leftarrow S \backslash\{f\}$

6: $\quad h \leftarrow$ the $\Pi$-reduction of $f$ with respect to $G$

7: $\quad$ if $h \neq 0$ then

8: $\quad G \leftarrow G \cup\{h\}$

9: $\quad S \leftarrow S \cup\left(\bigcup_{g \in G} S_{g, h}\right)$

10: $\quad$ end if

\section{1: end while}

This algorithm has been implemented for the particular case where $K[M]$ is a polynomial ring and $\Pi=\operatorname{Inc}(\mathbb{N})$ (i.e., the algorithm described in [Brouwer and Draisma 2011]) in the package Equivariant $G B$ [Hillar et al. 2013] for the computer algebra system Macaulay2 [Grayson and Stillman 2002]. When the algebra $K[M]$ is $\Pi$-Noetherian, termination of Algorithm 5.2 is guaranteed, but in general we cannot make this claim. 
We now turn our attention to the task of computing a finite $\operatorname{Inc}(\mathbb{N})$-generating set of binomials of a general toric map as in the main theorem. By the proof of Proposition 2.1 we may assume that $Y$ is as in (1), i.e., it consists of variables $y_{p, J}$ where $p$ runs through $[N]$ and $J$ runs through all $k_{p}$-tuples of distinct natural numbers. Section 2 then leads to the following analysis of this task.

Problem 5.3. Fix the names of algebras and maps in the following diagram:

$$
R[Y] \stackrel{\varphi}{\rightarrow} R[X] \stackrel{\psi}{\rightarrow} R[Z] .
$$

Here $\varphi$ is the map defined by (1), whose image is the $R$-algebra spanned by the matching monoid, and $\psi$ is any $\operatorname{Sym}(\mathbb{N})$-equivariant monomial map from $R[X]$ to $R\left[z_{i j} \mid i \in[k], j \in \mathbb{N}\right]$. For $\operatorname{ker}(\psi \circ \varphi)$, how does one compute

(a) a finite set of generators up to $\operatorname{Inc}(\mathbb{N})$-symmetry?

(b) a finite $\operatorname{Inc}(\mathbb{N})$-Gröbner basis with respect to a given $\operatorname{Inc}(\mathbb{N})$-compatible monomial order on $K[Y]$ ?

The algorithm we are about to construct solves Problem 5.3(a); indeed, we do not know whether a finite $\operatorname{Inc}(\mathbb{N})$-Gröbner basis as in part (b) exists! Our algorithm relies on the fact that we may replace $R[X]$ above by the matching monoid algebra $\operatorname{im} \varphi=R\left[x^{A} \mid A\right.$ good $]$, so as to get the sequence

$$
R[Y] \stackrel{\varphi}{\rightarrow} R\left[x^{A} \mid A \operatorname{good}\right] \stackrel{\psi}{\rightarrow} R[Z] .
$$

Most of our computations will take place in the ring $R\left[x^{A} \mid A \operatorname{good}\right][Z]$, which is itself a matching monoid with $N$ replaced by $N+k$ and $k_{p}=1$ for $p \in[N+k] \backslash[N]$. This monoid is Gröbner friendly by the following proposition.

Proposition 5.4. Let $M$ be a submonoid of $\mathbb{N}^{[k] \times \mathbb{N}}$ that is generated by the $\operatorname{Sym}(\mathbb{N})$ orbits of a finite number of matrices. For $\Pi=\operatorname{Inc}(\mathbb{N})$, the monoid algebra $K[M]$ satisfies EGB34.

Proof. Any such $K[M]$ is the image of some map $\varphi$ as in the main theorem (with $R=K)$, and so is $\operatorname{Inc}(\mathbb{N})$-Noetherian. Similarly $K\left[M^{3}\right]=K[M]^{\otimes 3}$ is $\operatorname{Inc}(\mathbb{N})$ Noetherian. For any $a, b \in M$, the monomial ideal $\left\langle T_{a, b}\right\rangle \subseteq K\left[M^{3}\right]$ is $\operatorname{Inc}(\mathbb{N})$-stable. Let $L \subseteq T_{a, b}$ be a minimal finite $\operatorname{Inc}(\mathbb{N})$-generating set of $\left\langle T_{a, b}\right\rangle$.

For any $(l, \pi a, \sigma b) \in T_{a, b}$, there is some $\left(m, a^{\prime}, b^{\prime}\right) \in L$ and $\tau \in \operatorname{Inc}(\mathbb{N})$ such that $\tau\left(m, a^{\prime}, b^{\prime}\right) \mid(l, \pi a, \sigma b)$. It is clear that $\tau a^{\prime}=\pi a$ and $\tau b^{\prime}=\sigma b$. Since $a^{\prime}$ and $b^{\prime}$ divide $m, \pi a$ and $\sigma b$ must divide $\tau m$, and in turn $\tau m$ divides $l$. But $l \in \operatorname{lcm}(\pi a, \sigma b)$ by assumption, so $l=\tau m$. Therefore $(l, \pi a, \sigma b)=\tau\left(m, a^{\prime}, b^{\prime}\right)$. This shows that $T_{a, b}$ is the union of the $\operatorname{Inc}(\mathbb{N})$-orbits of the elements of $L$, and then $L=O_{a, b}$.

To establish the same fact for a general pair $f, g \in K[M]$ we first determine $O_{a, b}$, where $a=\operatorname{lm}(f)$ and $b=\operatorname{lm}(g)$. For any $(l, \pi f, \sigma g) \in T_{f, g}$, the triple 
$(l, \pi a, \sigma b) \in T_{a, b}$ is in the orbit of some $\left(m, a^{\prime}, b^{\prime}\right) \in O_{a, b}$. This implies $a^{\prime}=\tau a$ for some $\tau \in \operatorname{Inc}(\mathbb{N})$, but $\tau$ is not unique. Define

$$
\Lambda_{a, a^{\prime}}:=\left\{\tau \in \operatorname{Inc}(\mathbb{N}) \mid a^{\prime}=\tau a ; \text { and } n \in \operatorname{im} \tau \text { for all } n>\ell_{a^{\prime}}\right\} .
$$

Here $\ell_{a^{\prime}}$ denotes the length of $a^{\prime}$ as in Section 4, the maximum index value among all nonzero columns of $a^{\prime}$. Note that $\Lambda_{a, a^{\prime}}$ is a finite set.

Since $\pi a$ is in the orbit of $a^{\prime}, \pi$ factors through some $\tau \in \Lambda_{a, a^{\prime}}$. So $(l, \pi f, \sigma g)=$ $\left(\gamma m, \alpha \tau_{1} f, \beta \tau_{2} g\right)$ for some $\gamma, \alpha, \beta \in \operatorname{Inc}(\mathbb{N}), \tau_{1} \in \Lambda_{a, a^{\prime}}$ and $\tau_{2} \in \Lambda_{b, b^{\prime}}$. Therefore

$$
T_{f, g} \subseteq \bigcup_{\left(m, f^{\prime}, g^{\prime}\right) \in U_{f, g}} \Pi m \times \Pi f^{\prime} \times \Pi g^{\prime}
$$

where

$$
U_{f, g}=\bigcup_{\left(m, a^{\prime}, b^{\prime}\right) \in T_{a, b}}\left\{\left(m, \tau_{1} f, \tau_{2} g\right) \mid \tau_{1} \in \Lambda_{a, a^{\prime}}, \tau_{2} \in \Lambda_{b, b^{\prime}}\right\}
$$

For each $\left(m, f^{\prime}, g^{\prime}\right)$, the set $\Pi m \times \Pi f^{\prime} \times \Pi g^{\prime}$ is the union of a finite number of Inc(N)-orbits. To prove this one can follow closely the proof of [Brouwer and Draisma 2011] Lemma 3.4. From the finite set of generators we select only those $\left(\gamma m, \alpha f^{\prime}, \beta g^{\prime}\right)$ with $\gamma m \in \operatorname{lcm}\left(\alpha f^{\prime}, \beta g^{\prime}\right)$, and call this set $O_{\left(m, f^{\prime}, g^{\prime}\right)}$. Then $O_{f, g}=\bigcup_{\left(m, f^{\prime}, g^{\prime}\right) \in U_{f, g}} O_{\left(m, f^{\prime}, g^{\prime}\right)}$ is as desired.

\section{Algorithm 5.5. $T=$ TORICIDEAL $(\varphi)$}

Require: $\varphi: R[Y] \rightarrow R[Z]$ is a monomial map as in the main theorem.

Ensure: $T$ is a finite set of generators of $\operatorname{ker} \varphi$ as $\operatorname{Inc}(\mathbb{N})$-stable ideal.

1: $\quad$ Replace $Y$ by the set of variables $\left\{y_{p, J}\right\}_{p, J}$ as in the proof of Proposition 2.1.

2: Decompose $\varphi$ with the composition of two maps $\varphi$ and $\psi$ as in diagram (2).

3: Consider the ideal $I_{\psi} \subset R\left[x^{A} \mid A\right.$ good $][Z]$ generated by the finite set $F$ of binomials $\psi\left(x^{A}\right)-x^{A}$, where $A \in \prod_{p \in[N]} \mathbb{N}^{\left[k_{p}\right] \times \mathbb{N}}$ is good of multidegree

$$
d \in\{(1,0, \ldots, 0), \ldots,(0,0,0, \ldots, 1)\} \subseteq \mathbb{N}^{[N]}
$$

and $\preceq$-minimal; the $\operatorname{Inc}(\mathbb{N})$-orbits of such monomials $x^{A}$ generate $R\left[x^{A} \mid\right.$ $A$ good].

4: Run Algorithm 5.2 for the input $F$ with respect to a monomial order that eliminates the variables $Z$. Since $R\left[x^{A} \mid A\right.$ good] $[Z]$ is the monoid algebra of a monoid where $\operatorname{Inc}(\mathbb{N})$-divisibility is a wpo, the algorithm terminates. Standard elimination theory implies that $G^{\prime}=G \cap R\left[x^{A} \mid A\right.$ good $]$ generates

$$
I_{\psi} \cap R\left[x^{A} \mid A \text { good }\right]=\operatorname{ker} \psi \cap \operatorname{im} \varphi .
$$

5: Let $T$ consist of preimages of elements in $G^{\prime}$ (one per element) and a finite number of binomials whose orbits generate $\operatorname{ker} \varphi$ (see Corollary 3.4). 
Remark 5.6. We can execute Algorithm 5.5 for any coefficient ring $R$ (not necessarily a field), since all polynomials that appear in the computation are binomials with coefficients \pm 1 .

In the following two remarks we comment on two major subroutines not spelled out in the sketch of the algorithm above.

Remark 5.7. Unlike in the usual Buchberger algorithm, the task of computing S-polynomials in Algorithm 5.2 is far from being trivial. To accomplish that, one needs to compute the set $O_{f, g}$, which can be done following the lines of the proof of Proposition 5.4. While this procedure is effective, by no means it is efficient.

Remark 5.8. In the last step of Algorithm 5.5 a preimage $\varphi^{-1}(g)$ of an element $g \in G$ can be computed by reducing the problem to one of computing maximal matchings of bipartite graphs, a well studied problem in combinatorics. Any monomial $x^{A} \in \operatorname{im} \varphi$ can be considered as a collection of $N$ bipartite graphs with adjacency matrices $A_{0}, \ldots, A_{N-1}$ as in Section 3, where each $A_{p}$ has bipartition $\left[k_{p}\right] \sqcup \mathbb{N}$. Fixing $A_{p}$, let $S \subset \mathbb{N}$ be the set of vertices in the second partition with degree $d_{A_{p}}$ (i.e., the indices of the columns of $A_{p}$ with column sum equal to $d_{A_{p}}$ ). A matching $B$ covering $\left[k_{p}\right]$ and $S$ can be computed using the Hungarian method or other algorithms for computing weighted bipartite matchings (see [Schrijver 2003, Chapter 17] for more details). The matching $B$ directly corresponds to a variable $y_{p, J} \in Y$ with $\varphi\left(y_{p, J}\right)=x^{B}$. Since $B$ covers $S$, it follows that $A_{p}-B$ is a good matrix. Therefore $x^{A_{p}} / \varphi\left(y_{p, J}\right)$ is also in $\operatorname{im} \varphi$ and can be decomposed further by repeating the process.

Algorithm 5.5 yields a solution to Problem 5.3(a) as an important theoretical consequence: a finite $\operatorname{Inc}(\mathbb{N})$-generating set of the toric ideals in the main theorem is computable. However, in view of Remark 5.7 and a more elementary procedure (albeit with no termination guarantee) given in the following section that solves a harder Problem 5.3(b) for a small example, we postpone a practical implementation of Algorithm 5.5.

\section{An example, and a more naïve implementation}

A more elementary approach to Problem 5.3 - indeed, to the hardest variant - is, for a given order on $[Y, Z]$, to directly apply the algorithm of [Brouwer and Draisma 2011] to the graph of the entire map $\psi \circ \varphi$, rather than computing generators for the kernels of $\psi$ and $\varphi$ separately as in Algorithm 5.5. The advantages of this approach are that it is simpler to implement, and that it produces not just a generating set, but an $\operatorname{Inc}(\mathbb{N})$-equivariant Gröbner basis. The disadvantage is that we do not know whether the procedure is guaranteed to terminate. We now set up a version of the 
usual equivariant Buchberger algorithm that is particularly easy to implement, and conclude with one nontrivial computational example.

For convenience let $\omega=\psi \circ \varphi$. Let $I_{\omega} \subset R[Y, Z]$ be the ideal corresponding to the graph of $\omega$, so $I_{\omega}$ is generated by the binomials of the form $y-\omega(y)$ for each variable $y \in Y$. Choosing a representative $y_{p}=y_{p,\left(0, \ldots, k_{p}-1\right)}$ of each $\operatorname{Sym}(\mathbb{N})$-orbit in $Y$, the ideal is $\operatorname{Inc}(\mathbb{N})$-generated by the finite set

$$
F:=\left\{\sigma y_{p}-\omega\left(\sigma y_{p}\right) \mid p \in[N], \sigma \in \operatorname{Sym}\left(\left[k_{p}\right]\right)\right\} .
$$

Choose an $\operatorname{Inc}(\mathbb{N})$-compatible monomial order $\leq$ on $R[Y, Z]$ that eliminates $Z$. Then apply to $F$ the equivariant Gröbner basis algorithm from [loc. cit.] (which is essentially Algorithm 5.2). Note that since we are working in a polynomial ring $R[Y, Z]$, rather than a more complicated monoid ring $R[X \mid X \operatorname{good}][Z]$, every pair of monomials has only one $\mathrm{lcm}$, which is straightforward to compute. If the procedure terminates with output $G$, then $G \cap R[Y]$ is an $\operatorname{Inc}(\mathbb{N})$-equivariant Gröbner basis of $I_{\omega} \cap R[Y]=\operatorname{ker} \omega$.

This procedure can be adapted to make use of existing, fast implementations of traditional Gröbner basis algorithms. For each $n \in \mathbb{N}$ truncate to the first $n$ index values by defining

$$
\begin{aligned}
& Y_{n}:=\left\{y_{p, J} \mid J \in[n]^{k_{p}}\right\}, \\
& Z_{n}:=\left\{z_{i, j} \in Z \mid j \in[n]\right\}, \\
& F_{n}:=\left\{y-\omega(y) \mid y \in Y_{n}\right\} .
\end{aligned}
$$

Let $I_{n}$ be the ideal in $R\left[Y_{n}, Z_{n}\right]$ generated by $F_{n}$. Each $I_{n}$ is Sym([n])-stable and $\bigcup_{n \in \mathbb{N}} I_{n}=I_{\omega}$. Let $\operatorname{Inc}(m, n)$ be the set of all strictly increasing maps $[m] \rightarrow[n]$, and equip $K\left[Y_{n}, Z_{n}\right]$ with the restriction of the $\operatorname{Inc}(\mathbb{N})$-monomial order $\leq$.

Algorithm 6.1. $G=$ TRUnCATEdBuchberger $(\omega)$

Require: $\varphi: R[Y] \rightarrow R[Z]$ is a monomial map in the main theorem.

Ensure: $G$ is an $\operatorname{Inc}(\mathbb{N})$-equivariant Gröbner basis of $\operatorname{ker} \varphi$.

$$
\begin{aligned}
& n \leftarrow \max _{p \in[N]} k_{p} \\
& \text { while true do } \\
& \quad F_{n} \leftarrow\left\{y-\omega(y) \mid y \in Y_{n}\right\} \\
& \quad G_{n} \leftarrow \operatorname{GRÖBNERBASIS}\left(F_{n}\right) \\
& m \leftarrow\lfloor(n+1) / 2\rfloor \\
& \text { if } m \geq \max _{p \in[N]} k_{p} \text { and } G_{n}=\operatorname{Inc}(m, n) G_{m} \text { then } \\
& \quad G \leftarrow G_{m} \cap R[Y] \\
& \quad \text { return } G \\
& \text { end if } \\
& n \leftarrow n+1 \\
& \text { end while }
\end{aligned}
$$


Here GRÖBNERBASIS denotes any algorithm to compute a traditional Gröbner basis. If TRUNCATEDBUCHBERGER $(\omega)$ terminates, this implies that there is some $m \geq \max _{p \in[N]} k_{p}$ such that $\operatorname{Inc}(m, n) G_{m}$ satisfies Buchberger's criterion for some $n \geq 2 m-1$. Then $G_{m}$ satisfies the equivariant Buchberger criterion, so $G_{m}$ is an equivariant Gröbner basis. Because we require that $m \geq \max _{p \in[N]} k_{p}$, the set $G_{m}$ generates $I_{\omega}$ up to $\operatorname{Inc}(\mathbb{N})$-action. Finally $G=G_{m} \cap R[Y]$ is an equivariant Gröbner basis for $\operatorname{ker} \omega$.

Example 6.2. Set $Y:=\left\{y_{j_{0}, j_{1}} \mid j_{0}, j_{1} \in \mathbb{N}, j_{0} \neq j_{1}\right\}$ and $Z:=\left\{z_{i} \mid i \in \mathbb{N}\right\}$, each consisting of a single $\operatorname{Sym}(\mathbb{N})$-orbit, and define the monomial map $\omega: R[Y] \rightarrow R[Z]$ by

$$
\omega: y_{j_{0}, j_{1}} \mapsto z_{j_{0}}^{2} z_{j_{1}}
$$

Whether $\operatorname{ker} \omega$ is finitely generated was posed as an open question in [Hillar and Martín del Campo 2013] (Remark 1.6). This is answered in the affirmative by Theorem 1.1, but by applying Algorithm 6.1 we have also explicitly computed an $\operatorname{Inc}(\mathbb{N})$-equivariant Gröbner basis. The Gröbner basis computations were carried out using the software package 4ti2 [Hemmecke et al. 2008], which features algorithms specifically designed for computing Gröbner bases of toric ideals. The monomial order on $Y$ is lexicographic, where variables are ordered by $y_{i, j}<y_{i^{\prime}, j^{\prime}}$ if $i<i^{\prime}$, or $i=i^{\prime}$ and $j<j^{\prime}$.

The result displayed in Table 1 consists of 51 generators with indices from $\{0,1,2,3,4,5\}$ and degrees up to 5 . Note that a minimal generating set resulting from a study of the family of equivariant toric maps of the form

$$
y_{i j} \mapsto z_{i}^{a} z_{j}^{b}, \quad i, j \in \mathbb{N}, i \neq j,
$$

for fixed $a, b \in \mathbb{N}$ in [Kahle et al. 2014] is much smaller.

Remark 6.3. As pointed out in the Introduction, the technique laid out in this article does not settle the question whether the finite generatedness of $\operatorname{ker} \varphi$ in the main theorem persists when $\operatorname{Inc}(\mathbb{N})$ acts with finitely many orbits on $Y$ and the monomial map $\varphi$ is required to be merely $\operatorname{Inc}(\mathbb{N})$-equivariant (though we do know that $\operatorname{im} \varphi$ needs not be $\operatorname{Inc}(\mathbb{N})$-Noetherian in this case).

However, a naïve elimination procedure terminates, for instance, for the $\operatorname{Inc}(\mathbb{N})$ analogue of Example 6.2, i.e., for the same map, but with the smaller set of variables

$$
Y:=\left\{y_{j_{0}, j_{1}} \mid j_{0}, j_{1} \in \mathbb{N}, j_{0}>j_{1}\right\}
$$

A computation that can be carried out with EquivariantGB [Hillar et al. 2013] produces a finite number of generators of the kernel:

$$
\left\{y_{3,1} y_{2,0}-y_{3,0} y_{2,1}, y_{3,2}^{2} y_{1,0}-y_{3,1} y_{3,0} y_{2,1}, y_{4,2} y_{3,2} y_{1,0}-y_{4,0} y_{3,1} y_{2,1}\right\} .
$$




$$
\begin{gathered}
\text { degree } 3 \\
\triangleleft y_{1,2} y_{0,1}^{2}-y_{1,0}^{2} y_{0,2} \triangleright \\
\triangleleft y_{2,0} y_{0,1}^{2}-y_{1,0} y_{0,2}^{2} \triangleright \\
y_{2,1} y_{0,2}^{2}-y_{2,0}^{2} y_{0,1} \\
\triangleleft y_{2,1} y_{1,0} y_{0,2}-y_{2,0} y_{1,2} y_{0,1} \triangleright \\
y_{2,1} y_{1,0}^{2}-y_{1,2}^{2} y_{0,1} \\
y_{2,1}^{2} y_{0,2}-y_{2,0}^{2} y_{1,2} \\
y_{2,1}^{2} y_{1,0}-y_{2,0} y_{1,2}^{2} \\
y_{2,1} y_{1,0} y_{0,3}-y_{2,0} y_{1,3} y_{0,1} \\
y_{2,1}^{2} y_{0,3}-y_{2,0}^{2} y_{1,3} \\
y_{2,3} y_{1,2} y_{0,2}-y_{2,0}^{2} y_{1,3} \\
y_{3,0} y_{1,2} y_{0,2}-y_{2,0} y_{1,3} y_{0,3} \\
y_{3,0} y_{1,2}^{2}-y_{2,0} y_{1,3}^{2} \\
y_{3,0} y_{2,1}^{2}-y_{2,3} y_{2,0} y_{1,3} \\
y_{3,1} y_{0,2}^{2}-y_{2,1} y_{0,3}^{2} \\
y_{3,1} y_{1,0} y_{0,2}-y_{3,0} y_{1,2} y_{0,1} \\
y_{3,1} y_{1,2} y_{0,2}-y_{2,1} y_{1,3} y_{0,3} \\
y_{3,1} y_{2,3} y_{0,3}-y_{3,0}^{2} y_{2,1} \\
y_{3,1}^{2} y_{0,2}-y_{3,0}^{2} y_{1,2} \\
y_{3,2} y_{1,3} y_{0,3}-y_{3,0}^{2} y_{1,2} \\
y_{3,2} y_{2,0} y_{1,3}-y_{3,0} y_{2,3} y_{1,2} \\
y_{3,2} y_{2,0} y_{1,4}-y_{3,0} y_{2,4} y_{1,2} \\
y_{3,2} y_{2,1} y_{0,3}-y_{3,1} y_{2,3} y_{0,2} \\
y_{3,2} y_{2,1} y_{0,4}-y_{3,1} y_{2,4} y_{0,2}-y_{4,1} y_{2,3} y_{0,2} \\
y_{4,0} y_{2,3} y_{1,3}-y_{3,0} y_{2,4} y_{1,4} \\
y_{4,1} y_{2,3} y_{0,3}-y_{3,1} y_{2,4} y_{0,4} \\
y_{4,2} y_{1,3} y_{0,3}-y_{3,2} y_{1,4} y_{0,4} \\
y_{4,0} y_{2,3} y_{1,2} \\
y_{2,3}
\end{gathered}
$$

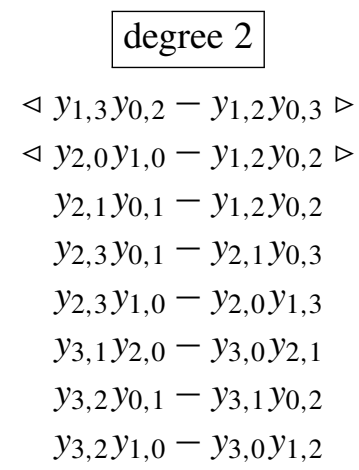

degree 4

$y_{2,1} y_{1,2} y_{0,3} y_{0,2}-y_{2,0}^{2} y_{1,3} y_{0,1}$ $y_{3,1} y_{2,3} y_{1,3} y_{0,4}-y_{3,0}^{2} y_{2,1} y_{1,4}$ $y_{3,1} y_{2,3}^{2} y_{0,4}-y_{3,0}^{2} y_{2,4} y_{2,1}$ $y_{3,2} y_{2,3} y_{1,3} y_{0,4}-y_{3,0}^{2} y_{2,4} y_{1,2}$ $y_{4,1} y_{2,3} y_{1,4} y_{0,4}-y_{4,0}^{2} y_{2,1} y_{1,3}$ $y_{4,1} y_{3,2} y_{1,4} y_{0,4}-y_{4,0}^{2} y_{3,1} y_{1,2}$ $y_{4,1} y_{3,4} y_{2,4} y_{0,5}-y_{4,0}^{2} y_{3,1} y_{2,5}$

$$
\begin{gathered}
\text { degree 5 } \\
y_{2,1} y_{1,2}^{2} y_{0,3}^{2}-y_{2,0}^{2} y_{1,3}^{2} y_{0,1} \\
y_{2,1} y_{1,2}^{2} y_{0,4} y_{0,3}-y_{2,0}^{2} y_{1,4} y_{1,3} y_{0,1} \\
y_{3,2} y_{2,3}^{2} y_{1,4} y_{0,4}-y_{3,0}^{2} y_{2,4}^{2} y_{1,2} \\
y_{3,2} y_{2,3}^{2} y_{1,4} y_{0,5}-y_{3,0}^{2} y_{2,5} y_{2,4} y_{1,2} \\
y_{4,1} y_{2,3} y_{1,4}^{2} y_{0,5}-y_{4,0}^{2} y_{2,1} y_{1,5} y_{1,3} \\
y_{4,1} y_{3,2} y_{1,4}^{2} y_{0,5}-y_{4,0}^{2} y_{3,1} y_{1,5} y_{1,2} \\
y_{4,3} y_{4,0}^{2} y_{3,2} y_{3,1}-y_{4,2} y_{4,1} y_{3,4}^{2} y_{0,3} \\
y_{5,1} y_{4,2} y_{3,5}^{2} y_{0,3}-y_{5,0}^{2} y_{4,3} y_{3,2} y_{3,1}
\end{gathered}
$$

Table 1. An Inc $(\mathbb{N})$-equivariant Gröbner basis for the kernel of $\omega$ in Example 6.2. The five highlighted binomials form a $\operatorname{Sym}(\mathbb{N})$ equivariant Markov basis according to [Kahle et al. 2014].

\section{Acknowledgments}

We thank a referee for pointing out the results of [Yamaguchi et al. 2014] on the Markov degree of the generalized Birkhoff model (which are stronger than a bound that we had in the original version of this paper) and for the remark that the integral polytopes capturing this model are not only normal but compressed (Remark 3.2). 


\section{References}

[Allman and Rhodes 2008] E. S. Allman and J. A. Rhodes, "Phylogenetic ideals and varieties for the general Markov model", Adv. in Appl. Math. 40:2 (2008), 127-148. MR 2008m:60145 Zbl 1131.92046

[Aschenbrenner and Hillar 2007] M. Aschenbrenner and C. J. Hillar, "Finite generation of symmetric ideals", Trans. Amer. Math. Soc. 359:11 (2007), 5171-5192. MR 2008g:13030 Zbl 1129.13008

[Brouwer and Draisma 2011] A. E. Brouwer and J. Draisma, "Equivariant Gröbner bases and the Gaussian two-factor model”, Math. Comp. 80:274 (2011), 1123-1133. MR 2012g:13049 Zbl 1211.13018

[Cohen 1987] D. E. Cohen, "Closure relations. Buchberger's algorithm, and polynomials in infinitely many variables", pp. 78-87 in Computation theory and logic, Lecture Notes in Comput. Sci. 270, Springer, Berlin, 1987. MR 89a:68113 Zbl 0641.68056

[Dickson 1913] L. E. Dickson, "Finiteness of the Odd Perfect and Primitive Abundant Numbers with $n$ Distinct Prime Factors", Amer. J. Math. 35:4 (1913), 413-422. MR 1506194 Zbl 44.0220 .02

[Draisma 2010] J. Draisma, "Finiteness for the $k$-factor model and chirality varieties", Adv. Math. 223:1 (2010), 243-256. MR 2011e:14091 Zbl 1211.13020

[Draisma and Eggermont 2015] J. Draisma and R. H. Eggermont, "Finiteness results for Abelian tree models", J. Eur. Math. Soc. (JEMS) 17:4 (2015), 711-738. MR 3336833

[Draisma and Kuttler 2009] J. Draisma and J. Kuttler, "On the ideals of equivariant tree models", Math. Ann. 344:3 (2009), 619-644. MR 2010e:62245

[Draisma and Kuttler 2014] J. Draisma and J. Kuttler, "Bounded-rank tensors are defined in bounded degree", Duke Math. J. 163:1 (2014), 35-63. MR 3161311 Zbl 1314.14109

[Grayson and Stillman 2002] D. R. Grayson and M. E. Stillman, "Macaulay2, a software system for research in algebraic geometry", 2002, http://www.math.uiuc.edu/Macaulay2/.

[Gross and Petrović 2013] E. Gross and S. Petrović, "Combinatorial degree bound for toric ideals of hypergraphs", Internat. J. Algebra Comput. 23:6 (2013), 1503-1520. MR 3109456 Zbl 1273.05237

[Haws et al. 2014] D. Haws, A. Martín del Campo, A. Takemura, and R. Yoshida, "Markov degree of the three-state toric homogeneous Markov chain model", Beitr. Algebra Geom. 55:1 (2014), 161-188. MR 3167787 Zbl 06299286

[Hemmecke et al. 2008] R. Hemmecke, R. Hemmecke, and P. Malkin, "4ti2 — A software package for algebraic, geometric and combinatorial problems on linear spaces", 2008, www.4ti2.de. Version 1.3.2.

[Higman 1952] G. Higman, "Ordering by divisibility in abstract algebras", Proc. London Math. Soc. (3) 2 (1952), 326-336. MR 14,238e Zbl 0047.03402

[Hillar and Martín del Campo 2013] C. J. Hillar and A. Martín del Campo, "Finiteness theorems and algorithms for permutation invariant chains of Laurent lattice ideals", J. Symbolic Comput. $\mathbf{5 0}$ (2013), 314-334. MR 2996883 Zbl 1263.13031

[Hillar and Sullivant 2012] C. J. Hillar and S. Sullivant, "Finite Gröbner bases in infinite dimensional polynomial rings and applications", Adv. Math. 229:1 (2012), 1-25. MR 2012k:16056 Zbl 1233.13012

[Hillar et al. 2013] C. Hillar, R. Krone, and A. Leykin, "EquivariantGB: Equivariant Gröbner bases in Macaulay2", 2013, http://people.math.gatech.edu/ rkrone3/EquivariantGB.html.

[Kahle et al. 2014] T. Kahle, R. Krone, and A. Leykin, "Equivariant lattice generators and Markov bases", pp. 264-271 in ISSAC 2014-Proceedings of the 39th International Symposium on Symbolic and Algebraic Computation, edited by K. Nabeshima, ACM, New York, 2014. MR 3239935 
[Kiraly and Rosen 2013] F. J. Kiraly and Z. Rosen, "Symmetries and finiteness for algebraic matroids", Poster at MEGA, 2013.

[Kruskal 1972] J. B. Kruskal, "The theory of well-quasi-ordering: A frequently discovered concept", J. Combinatorial Theory Ser. A 13 (1972), 297-305. MR 46 \#5184 Zbl 0244.06002

[de Loera et al. 1995] J. A. de Loera, B. Sturmfels, and R. R. Thomas, "Gröbner bases and triangulations of the second hypersimplex", Combinatorica 15:3 (1995), 409-424. MR 97b:13035 Zbl 0838.52015

[Nash-Williams 1963] C. S. J. A. Nash-Williams, “On well-quasi-ordering finite trees", Proc. Cambridge Philos. Soc. 59:04 (1963), 833-835. MR 27 \#3564 Zbl 0122.25001

[Norén 2015] P. Norén, “The three-state toric homogeneous Markov chain model has Markov degree two”, J. Symbolic Comput. 68:part 2 (2015), 285-296. MR 3283866 Zbl 1315.13045

[Ohsugi and Hibi 2001] H. Ohsugi and T. Hibi, "Convex polytopes all of whose reverse lexicographic initial ideals are squarefree", Proc. Amer. Math. Soc. 129:9 (2001), 2541-2546. MR 2002d:13037 Zbl 0984.13014

[Pachter and Sturmfels 2005] L. Pachter and B. Sturmfels (editors), Algebraic statistics for computational biology, Cambridge University Press, Cambridge, 2005. MR 2006i:92002 Zbl 1108.62118

[Schrijver 2003] A. Schrijver, Combinatorial optimization. Polyhedra and efficiency, Algorithms and Combinatorics 24, Springer, Berlin, 2003. MR 2004b:90004b Zbl 1041.90001

[Snowden 2013] A. Snowden, "Syzygies of Segre embeddings and $\Delta$-modules", Duke Math. J. 162:2 (2013), 225-277. MR 3018955 Zbl 1279.13024

[Sullivant 2006] S. Sullivant, "Compressed polytopes and statistical disclosure limitation”, Tohoku Math. J. (2) 58:3 (2006), 433-445. MR 2008g:52020 Zbl 1121.52028

[Yamaguchi et al. 2014] T. Yamaguchi, M. Ogawa, and A. Takemura, "Markov degree of the Birkhoff model”, J. Algebraic Combin. 40:1 (2014), 293-311. MR 3226827 Zbl 1302.62052

Communicated by Ravi Vakil

Received 2014-11-06 Revised 2015-03-06 Accepted 2015-06-12

j.draisma@tue.nl

Department of Mathematics and Computer Science, Technische Universiteit Eindhoven, P.O. Box 513, 5600 MB Eindhoven, Netherlands Vrije Universiteit and Centrum voor Wiskunde en Informatica, Amsterdam, Netherlands

r.h.eggermont@tue.nl

Department of Mathematics and Computer Science, Technische Universiteit Eindhoven, P.O. Box 513, 5600 MB Eindhoven, Netherlands

krone@math.gatech.edu School of Mathematics, Georgia Institute of Technology, 686 Cherry Street, Atlanta, GA 30332-0160, United States

leykin@math.gatech.edu School of Mathematics, Georgia Institute of Technology, 686 Cherry Street, Atlanta, GA 30332-0160, United States 


\section{Algebra \& Number Theory}

msp.org/ant

\section{EDITORS}

MANAGING EDITOR

Bjorn Poonen

Massachusetts Institute of Technology

Cambridge, USA

\author{
EDITORIAL BOARD CHAIR \\ David Eisenbud \\ University of California \\ Berkeley, USA
}

BOARD OF EDITORS

Georgia Benkart

Dave Benson

Richard E. Borcherds

John H. Coates

J-L. Colliot-Thélène

Brian D. Conrad

Hélène Esnault

Hubert Flenner

Sergey Fomin

Edward Frenkel

Andrew Granville

Joseph Gubeladze

Roger Heath-Brown

Craig Huneke

Kiran S. Kedlaya

János Kollár

Yuri Manin

Philippe Michel
University of Wisconsin, Madison, USA

University of Aberdeen, Scotland

University of California, Berkeley, USA

University of Cambridge, UK

CNRS, Université Paris-Sud, France

Stanford University, USA

Freie Universität Berlin, Germany

Ruhr-Universität, Germany

University of Michigan, USA

University of California, Berkeley, USA

Université de Montréal, Canada

San Francisco State University, USA

Oxford University, UK

University of Virginia, USA

Univ. of California, San Diego, USA

Princeton University, USA

Northwestern University, USA

École Polytechnique Fédérale de Lausanne
Susan Montgomery

Shigefumi Mori

Raman Parimala

Jonathan Pila

Anand Pillay

Victor Reiner

Peter Sarnak

Joseph H. Silverman

Michael Singer

Vasudevan Srinivas

J. Toby Stafford

Ravi Vakil

Michel van den Bergh

Marie-France Vignéras

Kei-Ichi Watanabe

Efim Zelmanov

Shou-Wu Zhang
University of Southern California, USA

RIMS, Kyoto University, Japan

Emory University, USA

University of Oxford, UK

University of Notre Dame, USA

University of Minnesota, USA

Princeton University, USA

Brown University, USA

North Carolina State University, USA

Tata Inst. of Fund. Research, India

University of Michigan, USA

Stanford University, USA

Hasselt University, Belgium

Université Paris VII, France

Nihon University, Japan

University of California, San Diego, USA

Princeton University, USA

\section{PRODUCTION}

production@msp.org

Silvio Levy, Scientific Editor

See inside back cover or msp.org/ant for submission instructions.

The subscription price for 2015 is US $\$ 255 /$ year for the electronic version, and $\$ 440 /$ year ( $+\$ 55$, if shipping outside the US) for print and electronic. Subscriptions, requests for back issues and changes of subscribers address should be sent to MSP.

Algebra \& Number Theory (ISSN 1944-7833 electronic, 1937-0652 printed) at Mathematical Sciences Publishers, 798 Evans Hall \#3840, c/o University of California, Berkeley, CA 94720-3840 is published continuously online. Periodical rate postage paid at Berkeley, CA 94704, and additional mailing offices.

ANT peer review and production are managed by EditFLOW ${ }^{\circledR}$ from MSP.

\section{PUBLISHED BY}

- mathematical sciences publishers

nonprofit scientific publishing

http://msp.org/

(C) 2015 Mathematical Sciences Publishers 


\section{Algebra \& Number Theory}

Volume $9 \quad$ No. $8 \quad 2015$

$G$-valued crystalline representations with minuscule $p$-adic Hodge type BRANDON LEVIN

Indicators of Tambara-Yamagami categories and Gauss sums

TATHAGATA BASAK and RYAN JOHNSON

The number of nonzero coefficients of modular forms $(\bmod p)$

JOËL BELLAÏCHE and KANNAN SOUNDARARAJAN

Noetherianity for infinite-dimensional toric varieties

JAN DRAISMA, Rob EgGERMOnT, ROBERT KRONE and ANTON LEYKIN

1881

On differential modules associated to de Rham representations in the imperfect residue field case

SHUN OHKUBO 